\title{
Topological Phases in the Single-Layer FeSe
}

\author{
Ningning $\mathrm{Hao}^{1,2}$ and Jiangping $\mathrm{Hu}^{1,2,3,4}$ \\ ${ }^{1}$ Beijing National Laboratory for Condensed Matter Physics and Institute of Physics, \\ Chinese Academy of Sciences, P.O. Box 603, Beijing 100190, China \\ ${ }^{2}$ Department of Physics, Purdue University, West Lafayette, Indiana 47907, USA \\ ${ }^{3}$ Department of Physics, Faculty of Science, King Abdulaziz University, Jeddah 21589, Saudi Arabia \\ ${ }^{4}$ Collaborative Innovation Center of Quantum Matter, Beijing, China
}

(Received 22 April 2014; published 24 September 2014)

\begin{abstract}
A distinct electronic structure was observed in the single-layer FeSe which shows surprisingly high-temperature superconductivity over $65 \mathrm{~K}$. Here, we demonstrate that the electronic structure can be explained by the effective strain effect due to substrates. More importantly, we find that this electronic structure can be tuned into robust topological phases from a topologically trivial metallic phase by the spinorbital interaction and couplings to substrates. The topological phase is robust against any perturbations that preserve the time-reversal symmetry. Our study suggests that nontrivial topology and high- $T_{c}$ superconductivity can be intertwined in the single FeSe layer to search novel physics.
\end{abstract}

DOI: 10.1103/PhysRevX.4.031053

\section{INTRODUCTION}

In the past several years, there have been rapid developments in the two research fields of condensed matter physics, iron-based high-temperature superconductors [1] and topological insulators [2,3]. The former includes many iron-pnictide and iron-chalcogenide materials in which the three-dimensional (3D) electrons of $\mathrm{Fe}$ atoms control electronic properties. The latter includes many semiconductors with strong spin-orbital couplings among $p$ orbital electrons. It is well known that the combination of topological properties with superconductivity can lead to many new physics, such as the creation of Majorana particles [2]. Normally, the combination is through a proximity effect by placing a topological insulator close to a conventional superconductor to induce superconductivity. However, the iron-based high-temperature superconductors are hardly candidates for such integration processes because they have short coherent length in the superconducting state. Thus, until now, these two fields have had little overlap.

The single-layer (SL) FeSe film [4-10] that is epitaxially grown on an $\mathrm{SrTiO}_{3}(001)$ surface exhibits several unique features compared with the bulk FeSe [11,12] and other Fe-based superconductors $[13,14]$. In the bulk FeSe, the superconducting transition temperature $T_{c} \sim 8 \mathrm{~K}$ [11], and the electronic structure is characterized by the presence of both hole pockets around the $\Gamma$ point and electron pockets around the M point in the first Brillouin zone(BZ) $[15,16]$.

Published by the American Physical Society under the terms of the Creative Commons Attribution 3.0 License. Further distribution of this work must maintain attribution to the author(s) and the published article's title, journal citation, and DOI.
Subject Areas: Condensed Matter Physics,

Topological Insulators

In the $\mathrm{SL} F \mathrm{FeSe}, T_{c}$ exceeding $65 \mathrm{~K}$ was observed [4]. Furthermore, there are only electron pockets around the M point, and the hole pockets at the $\Gamma$ point are absent [5-7]. The ARPES experiments $[5,6]$ have shown that the electronic structure of the SL FeSe cannot be obtained through the direct rigid band shifting because a new band gap below the Fermi surface is developed at the M point.

In this paper, we demonstrate that the SL FeSe is a potential candidate to integrate high- $T_{c}$ superconductivity together with topological properties. First, we discuss the origin of the novel band structure as a result of the lattice mismatch between the FeSe and substrate $\mathrm{SrTiO}_{3}$. We demonstrate that the lattice distortion can induce a phase transition around the $\mathrm{M}$ point from a gapless phase to a gapped phase and simultaneously suppress the holelike band at the $\Gamma$ point. Second, we find that the spin-orbital coupling can drive the SL FeSe from the trivial metal or semiconductor phase to a nontrivial topological insulating or metal phase. We calculate the phase diagram of the material in the presence of the spin-orbital coupling and the coupling to the substrate. As the gap at the M point is induced through coupling to the substrate so that the gap amplitude can be adjustable from zero to a finite value, a robust topological phase can always be achieved in the SL FeSe when the spinorbital coupling strength can overcome the gap. Our finding allows us to intertwine the nontrivial topology and high- $T_{c}$ superconductivity within one material.

\section{MODEL FOR THE BAND STRUCTURE OF THE SINGLE-LAYER FeSe}

In this section, we discuss the origin of the novel band structure as a result of the lattice mismatch between the FeSe and substrate $\mathrm{SrTiO}_{3}$, and we demonstrate that the 
lattice distortion can induce a phase transition around the M point from a gapless phase to a gapped phase and simultaneously suppress the holelike band at the $\Gamma$ point.

It is known that the electronic structure in the bulk FeSe is two dimensional and determined by the single-FeSe trilayer structure, as shown in Figs. 1(a) and 1(b) in which the unit cell includes two $\mathrm{Fe}$, labeled as $A$ and $B$, and two Se. The band-structure calculations from the density functional theory (DFT) show that only the five 3D orbitals of Fe play significant roles near Fermi surfaces [15]. A general effective $d$-orbital model for the band structure in real space can be written as

$$
H_{t}=\sum_{\sigma} \sum_{m n} \sum_{i j}\left(t_{i j}^{m n}+\epsilon_{m} \delta_{m n} \delta_{i j}\right) d_{m, \sigma}^{\dagger}(i) d_{n, \sigma}(j) .
$$

Here, $\sigma$ labels the spin; $m, n$ label five $d$ orbitals: $\left(d_{x z}, d_{y z}, d_{x^{2}-y^{2}}, d_{x y}, d_{z^{2}}\right) ; i, j$ label lattice sites; $t_{i j}^{m n}$ are the corresponding hopping parameters; $\epsilon_{m}$ is the on-site energy

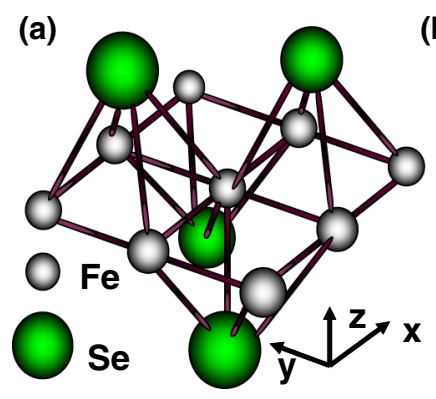

(b)
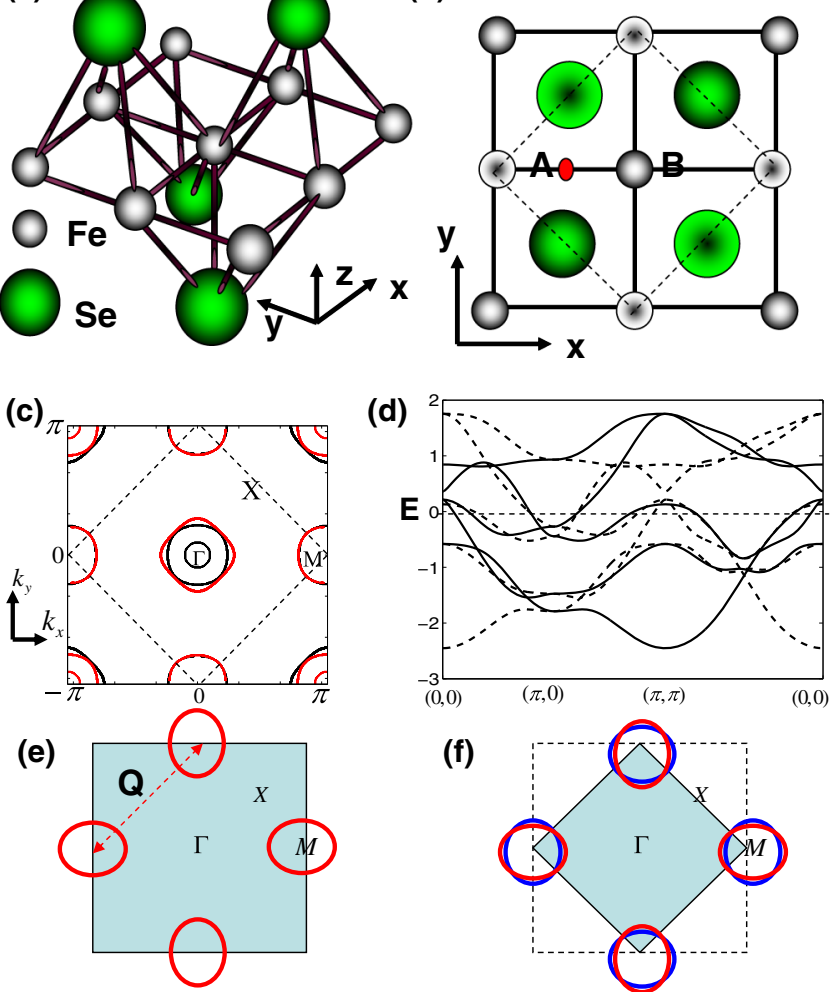

(f)

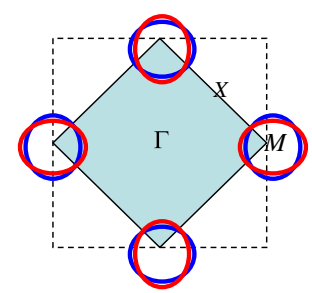

FIG. 1. (a) The lattice structure of FeSe. (b) The top view of (a). The two-Fe unit cell is enclosed by the dashed lines, with the two-Fe sublattice labeled with $\mathrm{A}$ and $\mathrm{B}$. The inverse center is labeled by the red elliptic spot at the midpoint of the A-B link. (c) The Fermi surface for the bulk FeSe, with three hole pockets located around the $\Gamma$ point and two electron pockets around the $\mathrm{M}$ point in the two-Fe BZ. The two-Fe BZ is enclosed by the dashed lines. (d) The band structure along high-symmetry lines. "(e), (f)" The schematic Fermi surface for SL FeSe is shown in the one-Fe and two-Fe unit-cell picture. The shaded region is the corresponding BZ. In (e), $Q=(\pi, \pi)$ is the folding wave vector. of the $d$ orbital; and $d_{m, \sigma}^{\dagger}(i)$ creates a spin- $\sigma$ electron in the $m$ th orbital of $\mathrm{Fe}$ at site $i$. In the momentum space, the Hamiltonian can be written as

$$
H_{t}=\sum_{k} \phi^{\dagger}(k) A(k) \phi(k)+\sum_{k^{\prime}} \phi^{\dagger}\left(k^{\prime}\right) A\left(k^{\prime}\right) \phi\left(k^{\prime}\right) .
$$

Here, $k$ is defined in the $\mathrm{BZ}$ of the one-Fe unit cell, and $k^{\prime}=k+Q$ with $Q=(\pi, \pi) . \phi(k)=\left[d_{x z}(k), d_{y z}(k), d_{x^{2}-y^{2}}(k)\right.$, $\left.d_{x y}(k), d_{z^{2}}(k)\right]^{T} . A(k)$ has been widely utilized to describe the electronic structure of all kinds of iron-based superconductors $[17,18]$. The difference between the one-Fe unit cell and the two-Fe unit cell has been discussed in detail $[17,19]$. Theoretically, the effective one-Fe unit-cell picture is usually used for simplicity, and the explicit form of $A(k)$ is presented in Appendix A.

The Fermi surface and band structure along highsymmetry lines are shown in Figs. 1(c) and 1(d). In Fig. 1(c), there are three hole pockets around the $\Gamma$ point and two electron pockets around the $\mathrm{M}$ point. We have specified that the black pockets in Fig. 1(c) are from $A(k)$, while the red pockets are from $A\left(k^{\prime}\right)$. In Fig. 1(d), the solid or dashed lines denote the bands from $A(k)$ or $A\left(k^{\prime}\right)$.

In Fig. 1, there is no gap opening in the band structure at the $M$ point. To gain the insight of the gap opening observed experimentally in the SL FeSe, we analyze the symmetry characters of the bands. As shown in Fig. 1, there are four bands along the $\Gamma$-M direction near Fermi surfaces. Each $A(k)$ and $A\left(k^{\prime}\right)$ contribute two bands. The bands contributed from $A(k)$ and $A\left(k^{\prime}\right)$ have opposite parity $[19,20]$. The two bands from $A(k)$ belong to $A_{2}$ and $B_{2}$ representations [21], which is the reason why the two bands can cross each other without showing the sign of hybridization. However, the two bands from $A\left(k^{\prime}\right)$ belong to the same $B_{1}$ representations [21]. If these two bands cross each other, the hybridization must arise. We notice that these two bands mainly have $d_{x z}$ and $d_{x y}$ orbital characters, respectively, near the M point. Moreover, the symmetries of the bands do not depend on the interorbital couplings because the couplings between two different $d$ orbitals at highsymmetry points, such as $\Gamma$ and $M$, vanish. Therefore, we can adjust the relative energy difference between $d_{x z}$ and $d_{x y}$ at M points to create a crossing between the two bands. Such a crossing can result in a gap opening at the M point because of the hybridization.

To confirm the above symmetric analysis, we present an evolution of the band structure by manipulating hopping parameters in Fig. 2. The bulk band structures are shown in Figs. 2(a) and 2(d). Note that we set all interorbital hoppings to zero in Fig. 2(a) in order to clearly distinguish the five $d$-orbital bands. In Fig. 2(a), the red-dashed $d_{x z}$ band and the blue-dashed $d_{x y}$ band have no crossing along the $(0,0)-(\pi, 0)$ line. In Fig. 2(d), there is no band-gap opening around the $\mathrm{M}$ point, i.e., $(\pi, 0)$ [see the red rectangle region in Fig. 2(d)]. When we adjust the values of some hopping parameters (see the caption of Fig. 2), the 

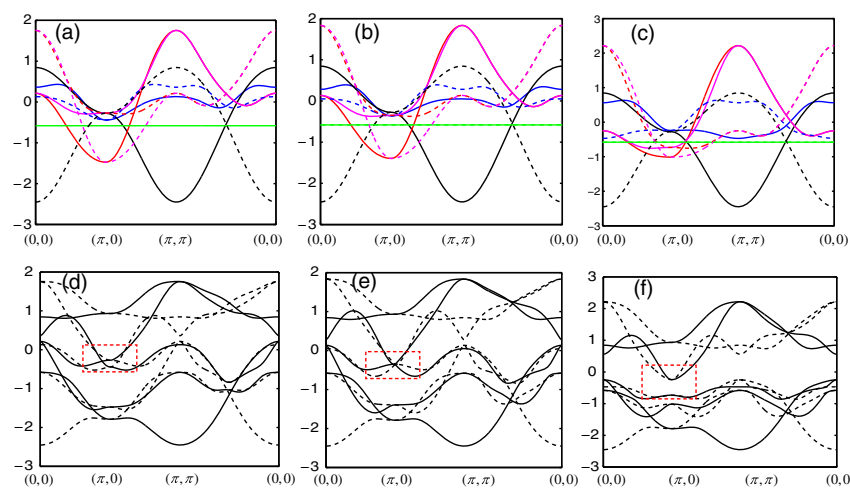

FIG. 2. (a)-(c) The band structures along high-symmetry lines for the case without interorbital hoppings. (a) The bulk case. (b) The adjusted parameters $t_{x y}^{44}=0.066, t_{x}^{14}=0.405$, and $t_{x}^{11}=-0.120$. (c) $t_{x y}^{44}=0.036, t_{x}^{44}=0.163, t_{x}^{14}=0.405$, and $t_{x}^{11}=-0.311$. The solid and dashed lines are from $A(k) / A(k+Q)$. The red, magenta, blue, black, and green lines are the $d_{x z} / d_{y z} / d_{x y} / d_{x^{2}-y^{2}} / d_{z^{2}}$ bands. (d)-(f) The cases with interorbital hoppings, corresponding to (a)-(c).

red-dashed $d_{x z}$ band is pushed down and the blue-dashed $d_{x y}$ band is pushed up. The critical case is shown in Fig. 2(b); the two bands meet each other at the M point. Then, a further push leads to the crossing between reddashed $d_{x z}$ and blue-dashed $d_{x y}$ bands at some point between $(0,0)$ and $(\pi, 0)$ [see Fig. 2(c)]. Turning on the interorbital hoppings, we obtain a new band structure shown in Fig. 2(f). Compared with the bulk bands in Fig. 2(d), the bands in Fig. 2(f) present some new features, such as the following: (1) The hole pockets around the $\Gamma$ point are pushed down the Fermi energy, with only electron pockets around the M point surviving, (2) the band top of the hole pockets has nearly the same energy as the band bottom of the electron pockets, and (3) there is a band gap opened at the $\mathrm{M}$ point [see the red rectangle region in Fig. 2(f)]. These features, as shown in Fig. 2(f), are comparable to the ARPES observations [5,6].

From Fig. 2, we can find that the evolution of the band structure from bulk FeSe to a SL FeSe is strongly sensitive to the change of a single hopping parameter $t_{x}^{11}$; i.e., the amplitude of intra- $d_{x z} / d_{y z}$ hopping along the $x / y$ directions. Namely, the nearest-neighbor intraorbital hoppings for $d_{x z}$ and $d_{y z}$ changes from the strong anisotropy in the bulk FeSe to near isotropy in the SL FeSe. We argue that this is the essential reason to drive the electronic structure of the SL FeSe. The SL FeSe film is epitaxially grown on an $\mathrm{SrTiO}_{3}(001)$ surface. Consequently, the lattice constant for the SL FeSe film should match the lattice constant for $\mathrm{SrTiO}_{3}$, i.e., $3.905 \AA$ A. Compared with the bulk FeSe with the lattice constant $3.76 \AA$ [11], the apparent lattice mismatch between FeSe and $\mathrm{SrTiO} 3$ should exert a strong tensile strain on the FeSe film and drive the lattice distortion for the FeSe film [7,8]. The lattice distortion naturally induces the change of hopping. To show the effect of the lattice distortion on hopping parameters, we have used the Slater-Koster method [22] to calculate the overlap integrals between different orbitals. The detailed derivations and calculations are presented in Appendix B. We find that the $t_{x}^{11}$ are strongly affected by the lattice distortion, and other hoppings are weakly adjusted. This is consistent with our above analysis.

\section{FULL HAMILTONIAN AND THE SPIN-ORBITAL COUPLING IN THE SL FeSe}

After obtaining the new band structure, we can ask whether the new band gap at the $M$ point [see the red rectangle region in Fig. 2(f)] is topologically trivial or nontrivial when the spin-orbital coupling (SOC) is considered. As the $t_{2 g}$ orbitals are significant near the Fermi surface, we can write the SOC within the $t_{2 g}$ subset. Up to the next-nearest neighbor, the general SOC Hamiltonian in momentum space can be written as

$$
\begin{aligned}
H_{s o}= & H_{s o 1}+H_{s o 2}, \\
H_{s o 1}= & \sum_{\tilde{k}=k, k^{\prime}}(-1)^{\sigma} \lambda_{\perp}(\tilde{k}) d_{x z, \sigma}^{\dagger}(\tilde{k}) d_{y z, \sigma}(\tilde{k})+\text { H.c., } \\
H_{s o 2}= & \sum_{\tilde{k}=k, k^{\prime}}\left[i \lambda_{\|, x z}(\tilde{k}) d_{x z, \uparrow}^{\dagger}(\tilde{k})+\lambda_{\|, y z}(\tilde{k}) d_{y z, \uparrow}^{\dagger}(\tilde{k})\right] d_{x y, \downarrow}(\tilde{k}+Q) \\
& -\sum_{\tilde{k}=k, k^{\prime}} d_{x y, \uparrow}^{\dagger}(\tilde{k})\left[i \lambda_{\|, x z}(\tilde{k}) d_{x z, \downarrow}(\tilde{k}+Q)\right. \\
& \left.+\lambda_{\|, y z}(\tilde{k}) d_{y z, \downarrow}(\tilde{k}+Q)\right]+ \text { H.c. }
\end{aligned}
$$

Here, $\quad \sigma=\mp \quad$ for $\quad$ spin $\uparrow \quad$ or $\quad \downarrow, \quad \lambda_{\perp}(\tilde{k})=\left(\lambda_{\perp}^{o}+\right.$ $\left.4 \lambda_{\perp}^{n n} \cos \tilde{k}_{x} \cos \tilde{k}_{y}\right)$, and $\lambda_{\|, x z / y z}(\tilde{k})=\lambda_{\|}^{o}$. The index $\alpha=$ $o, n n$ in the $\lambda_{\|, \perp}^{\alpha}$ indicates the on-site and next-nearestneighbor SOC, respectively. $H_{s o 1}$ describes the SOC between $d_{x z}$ and $d_{y z}$ orbitals. This term does not flip spin; it conserves momentum with respect to the one-Fe unit cell. $H_{s o 2}$ describes the SOC between $d_{x y}$ and $d_{x z, y z}$. This term flips spin and does not conserve momentum with respect to the one-Fe unit cell. Therefore, $H_{s o 2}$ essentially breaks the nonsymmorphic lattice symmetry in the SL FeSe, namely, a one-unit translation along the Fe-Fe direction followed by a mirror reflection with respect to the layer [23]. In the presence of $H_{s o 2}$, the two-Fe unit cell cannot be reduced to the one-Fe unit cell.

The $H_{s o 2}$ is not the only term that breaks the reduction to the one-Fe unit cell. If we consider the effect of the substrate, the space inversion symmetry is naturally broken for the SL FeSe. Such a parity breaking can also result in a term that only preserves the two-Fe unit cell [20],

$$
H_{s}=\sum_{m, \sigma} \sum_{\tilde{k}=k, k^{\prime}} \xi_{s}(\tilde{k}) d_{m, \sigma}^{\dagger}(\tilde{k}) d_{m, \sigma}(\tilde{k}+Q) .
$$

Here, $\xi_{s}(\tilde{k})$ measures this parity-breaking effect. Likewise, we only focus on three $t_{2 g}$ orbitals. If we take a constant 
$\xi_{s}(\tilde{k})$, the term describes a staggered potential in the iron square lattice.

The full Hamiltonian for the electronic structure of the SL FeSe with a substrate is a combination of $H_{t}, H_{s o}$, and $H_{s}$, namely,

$$
H=H_{t}+H_{s o 1}+H_{s o 2}+H_{s} .
$$

The last two terms in $H$ only preserve the translational symmetry with respect to the two-Fe unit cell.

\section{TOPOLOGICAL PHASES AND EFFECTIVE HAMILTONIAN}

We first concentrate on the effect of $H_{s o 1}$ which preserves the one-Fe unit cell. Namely, we ignore both $H_{s o 2}$ and $H_{s}$. The glancing features can be revealed through the numerical results of the band structure of the Hamiltonian $H=H_{t}+H_{s o 1}$. The results are shown in Fig. 3. In Figs. 3(a)-3(c), it is explicitly shown that the band gap undergoes a closing and reopening process at the $\mathrm{M}$ point when $\lambda_{\perp}^{o}$ and $\lambda_{\perp}^{n n}$ are tuned from zero to some finite values. Such a phenomenon is a strong indication of a topological phase transition [24,25]. Figures 3(d)-3(i) provide a clear proof of the occurrence of the topological phase transition. The spectra in Figs. 3(d)-(f) and Figs. 3(g)-(i) are plotted within the one-Fe unit cell and the two-Fe unit cell, respectively. The latter can be obtained through folding the one-Fe unit-cell picture with the folding wave vector $Q=(\pi, \pi)$ shown in Fig. 1(e). It is clear that the signature of the topological phase, gapless edge states, emerges after the reopening of the band gap.

We can also construct an effective Hamiltonian to describe the topological transition. We define the new

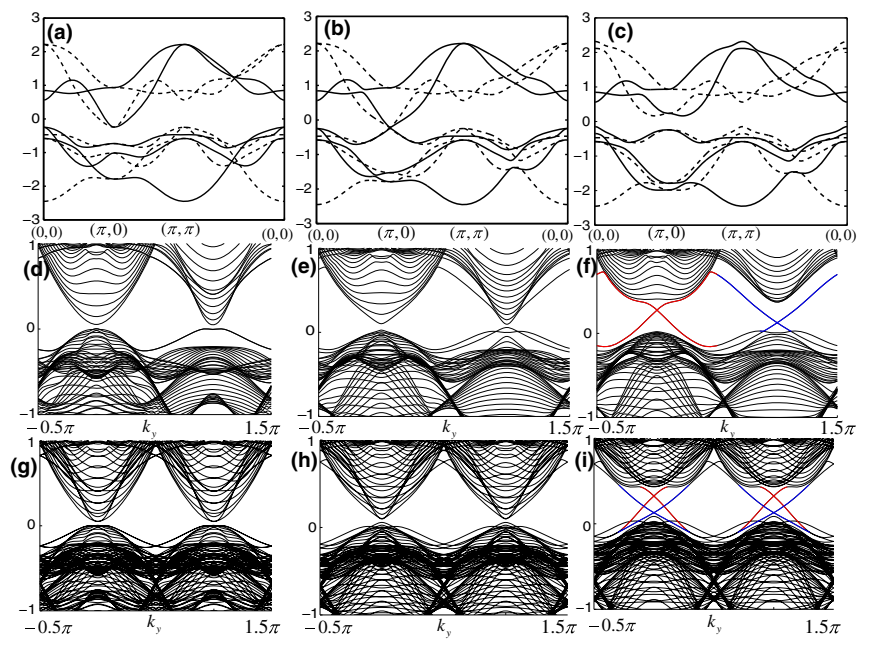

FIG. 3. (a)-(c) The spectrum along high-symmetry lines for different SOC parameters with (a) $\lambda_{\perp}^{o}=0, \quad \lambda_{\perp}^{n n}=0$, (b) $\lambda_{\perp}^{o}=0.32, \lambda_{\perp}^{n n}=0.08$, and (c) $\lambda_{\perp}^{o}=0.5, \lambda_{\perp}^{n n}=0.12$. Other parameters $\lambda_{\|}^{o}$ and $\xi_{s}$ are set to zero. (d)-(f) [(g)-(i)] are the corresponding one-Fe unit-cell (two-Fe unit-cell) edge spectrum with open boundary along the $x$ direction. The width of the single-layer film is 21 in units of the lattice constant. basis for $d_{x z}$ and $d_{y z}$ orbitals according to the eigenstates of azimuthal quantum number $l=2$ and magnetic quantum number $m= \pm 1$, i.e., $d_{(2,1), \sigma}(\tilde{k})=-(1 / \sqrt{2})\left[d_{x z, \sigma}(\tilde{k})+\right.$ $\left.i d_{y z, \sigma}(\tilde{k})\right]$ and $d_{(2,-1), \sigma}(\tilde{k})=(1 / \sqrt{2})\left[d_{x z, \sigma}(\tilde{k})-i d_{y z, \sigma}(\tilde{k})\right]$. Around each $\mathrm{M}$ point, the band structure can be spanned by $\quad \Psi_{\mathrm{eff}}(\tilde{k})=\left[\phi_{\mathrm{eff}, \uparrow}(\tilde{k}), \phi_{\mathrm{eff}, \downarrow}(\tilde{k})\right]^{T}, \quad$ with $\quad \phi_{\mathrm{eff}, \sigma}(\tilde{k})=$ $\left[d_{x y, \sigma}(\tilde{k}), d_{\left(2,-(-1)^{\sigma}\right), \sigma}(\tilde{k})\right]^{T}$, and it is described by the effective Hamiltonian

$$
H_{\mathrm{eff}}=\sum_{\tilde{k}=k, k^{\prime}} \Psi_{\mathrm{eff}}^{\dagger}(\tilde{k}) H_{\mathrm{eff}}(\tilde{k}) \Psi_{\mathrm{eff}}(\tilde{k})
$$

where

$$
H_{\mathrm{eff}}(\tilde{k})=\sum_{a=0}^{5} \varepsilon_{a}(\tilde{k}) \Gamma^{a}+\sum_{a<b=1}^{5} \varepsilon_{a b}(\tilde{k}) \Gamma^{a b}
$$

is a $4 \times 4$ matrix. The $\Gamma$ matrices are defined as $\Gamma^{(0,1,2,3,4,5)}=\left(\tau^{0} \otimes s^{0}, \tau^{0} \otimes s^{z}, \tau^{0} \otimes s^{y}, \tau^{z} \otimes s^{x}, \tau^{y} \otimes s^{x}\right.$, $\tau^{x} \otimes s^{x}$ ), where the Pauli matrices $\tau$ and $s$ span the orbital and spin subspaces, and $\Gamma^{a b}=\left[\Gamma^{a}, \Gamma^{b}\right] /(2 i)$. The nonzero elements are $\varepsilon_{0 / 1}(\tilde{k})=(1 / 2)\left[E_{x y}(\tilde{k}) \pm E_{(2,-1)}(\tilde{k}) \pm\left|\lambda_{\perp}(\tilde{k})\right|\right]$ and $\varepsilon_{12 / 13}(\tilde{k})= \pm \sqrt{2} t_{x}^{14} \sin \tilde{k}_{y / x}$, in which $E_{x y}(\tilde{k})=A_{44}(\tilde{k})$ and $E_{(2,-1)}(\tilde{k})=(1 / 2)\left[A_{11}(\tilde{k})+A_{22}(\tilde{k})\right]$.

The above effective Hamiltonian has the same form as that for HgTe quantum wells [25]. At each M point, the band gap of Eq. (9) is measured by a Dirac mass $\varepsilon_{1}(\tilde{k})$. When the $\lambda_{\perp}(\tilde{k})$ overcomes the trivial band gap measured by $E_{x y}(\tilde{k})-E_{(2,-1)}(\tilde{k})$, namely, when the mass $\varepsilon_{1}(\tilde{k})<0$ changes its sign from positive to negative, a trivial to nontrivial topological phase transition arises.

We notice that there are two nontrivial Dirac cone structures without considering the spin degree of freedom in Hamiltonian $H=H_{t}+H_{s o 1}$ as indicated by the edge states with red and blue colors in Fig. 3(i). This is because the Hamiltonian preserves the full nonsymmorphic lattice symmetry so that the band structure decouples into two independent parts in view of the two-Fe unit cell. In the two-Fe unit-cell BZ, there are two nontrivial Dirac cone structures at each BZ zone corner, with one for each part. If we understand this in the one-Fe unit-cell picture, only one nontrivial Dirac cone structure exists at each $M$ point of the one-Fe BZ, as indicated by the edge states with red or blue colors in Fig. 3(f), and this nontrivial Dirac cone structure can be characterized by a $Z_{2}$ time-reversal invariant topological number when the spin degree of freedom is taken into account $[24,25]$. However, with even (two) nontrivial Dirac cone structures in the view of 2-Fe unit-cell picture as indicated by the edge states with red and blue colors in Fig. 3(i), the topological phase generally is unstable. One can imagine that any perturbations that break nonsymmorphic lattice symmetry may lead to a coupling between the two nontrivial Dirac cone structures, and this coupling opens a gap between the relevant edge 
states with red and blue colors shown in Fig. 3(i). Therefore, we call this topological phase the weak topological phase [26].

As we mentioned earlier, in the general Hamiltonian [Eq. (7)] for the SL FeSe with a substrate, both $H_{s o 2}$ and $H_{s}$ break the nonsymmorphic lattice symmetry. Therefore, we have to determine how these two terms affect the weak topological phase. First, we consider the effect of $H_{s o 2}$. Indeed, the spin-flip term in $H_{s o 2}$ induces the couplings between the two Dirac cones to create a gap on the edge states. If we assume that the SOC parameter $\lambda_{\|}^{o}$ is small, the gap that is open on the edge states is given by $\sim\left|\lambda_{\|}^{o}\right|^{2} /\left|t_{x}^{14}\right|$. Therefore, in principle, the $\lambda_{\|}^{o}$ term in Eq. (5) can be considered as a controlling parameter of the gap. The situation is very similar to a topological crystalline insulator

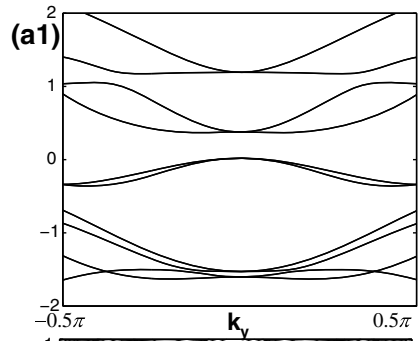

(a2)
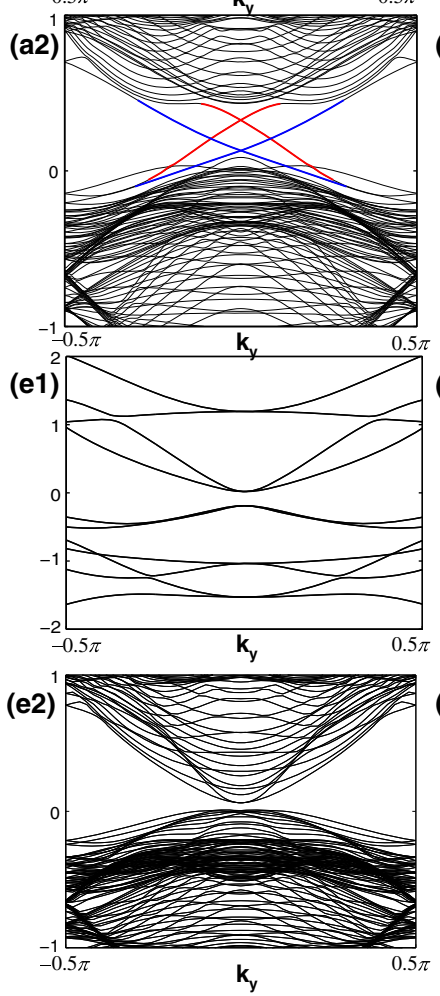

(b1)

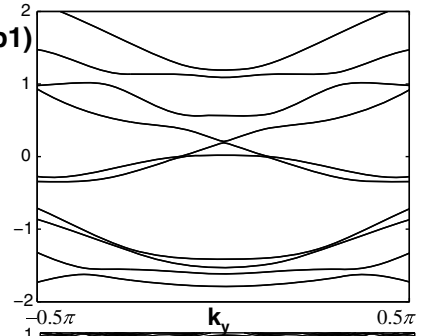

(b2)
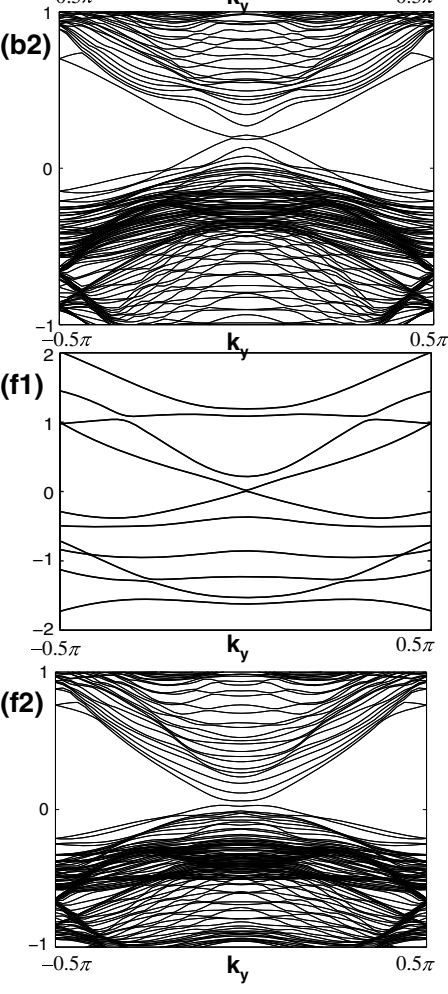

$[27,28]$ and the topological phase in a system with a nonsymmorphic lattice symmetry [29].

However, if we turn on $H_{s}$, the situation is drastically different. Rather than destroying the topological phase, we find that $H_{s}$ can stabilize the topological phase and that it drives the system to a strong topological phase. To understand it, we consider the effective Hamiltonian in Eq. (8) describing the weak topological phase. If we add $H_{s}$, for $\lambda_{\perp}(k)>0$, the spectrum becomes $E(k)= \pm \sqrt{\epsilon_{12}^{2}(k)+\epsilon_{13}^{2}(k)+\left[\epsilon_{1}(k) \pm \xi_{s}\right]^{2}}$. We can find that the effect of $H_{s}$ is to change the Dirac masses at the two $\mathrm{M}$ points. The changes, $\varepsilon_{1}(k) \pm \xi_{s}$, are different for the Dirac cones at the two different M points. Therefore, $H_{s}$ can create a band inversion in one Dirac cone but not the other, a case for a strong topological phase with an odd number

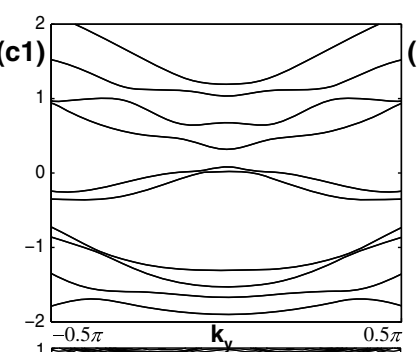

(c2)
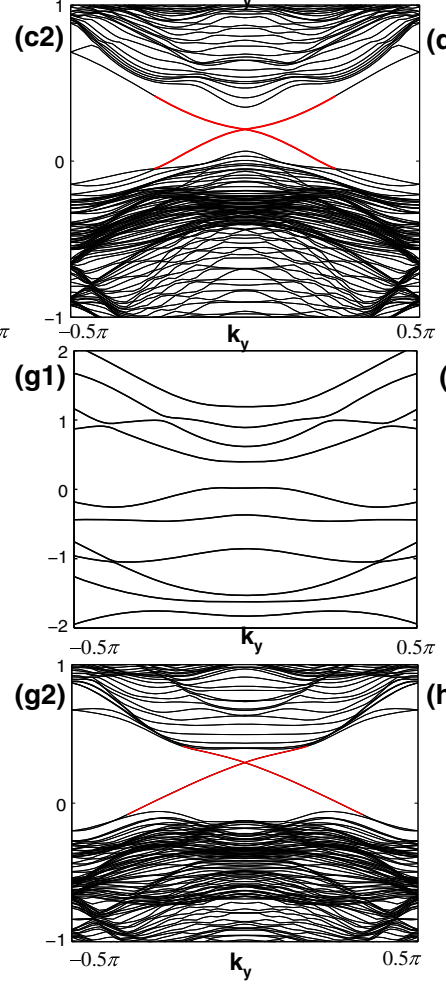

(d1)

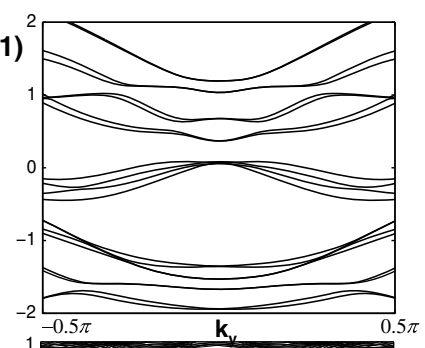

(d2)

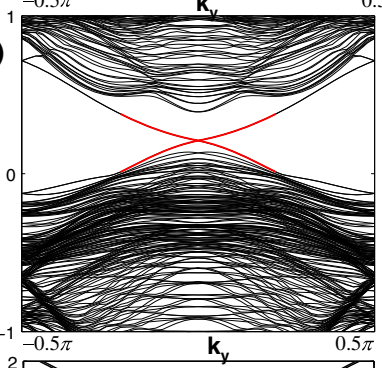

(h1)
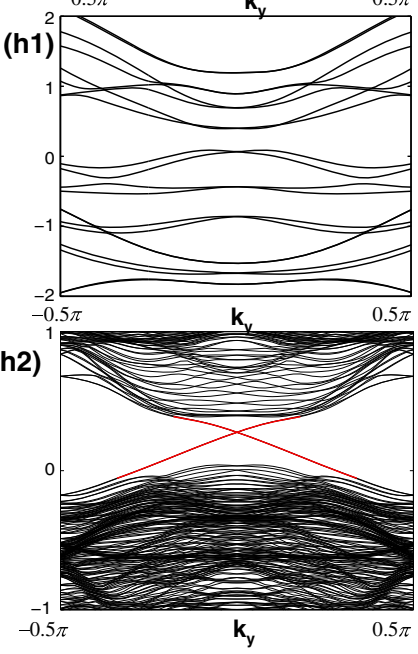

FIG. 4. (a1)-(d1) The evolution of the spectrum with $k_{x}=\pi, k_{y} \in[-(\pi / 2),(\pi / 2)]$ for parameters $\left(\lambda_{\perp}^{o}, \lambda_{\perp}^{n n}, \lambda_{\|}^{o}, \xi_{s}\right)$ with the values $(0.5,-0.12,0,0)$ in (a1), $(0.5,-0.12,0,0.19)$ in (b1), $(0.5,-0.12,0,0.3)$ in $(\mathrm{c} 1)$, and $(0.5,-0.12,0.2,0.3)$ in $(\mathrm{d} 1)$. (a2) $-(\mathrm{d} 2)$ The corresponding edge spectrum with an open boundary along the $x$ direction. (a2) is the weak topological phase, and (c2) and (d2) are the strong topological phase. (b2) is the critical point. (e1)-(h1) The evolution of the spectrum with $k_{x}=\pi, k_{y} \in[-(\pi / 2),(\pi / 2)]$ for parameters $\left(\lambda_{\perp}^{o}, \lambda_{\perp}^{n n}, \lambda_{\|}^{o}, \xi_{s}\right)$ with the values $(0.2,-0.05,0,0)$ in $(\mathrm{e} 1),(0.2,-0.05,0,0.2)$ in (f1), $(0.2,-0.05,0,0.6)$ in (g1), and $(0.2,-0.05,0.2,0.6)$ in (h1). (e2)-(h2) are the corresponding edge spectra with an open boundary along the $x$ direction. (e2) is the trivial phase, and (g2) and (h2) are the strong topological phase. (f2) is the critical point. In (a2)-(d2) and (e2)-(h2), the width of the single-layer film is 21 units of Fe-Fe lattice constants. 
of nontrivial Dirac cones. Thus, the strong topological phase is robust against any non-time-reverse-symmetry broken couplings, including the $H_{s o 2}$, as long as the coupling does not close the bulk energy gap.

The numerical proof of the above analysis is plotted in Fig. 4 in which the edge spectra in Figs. 4(d2) and 4(h2) clearly indicate a strong topological phase. More specifically, we discuss the strong topological phases in two cases: (1) $\varepsilon_{1}(k)<0$ and (2) $\varepsilon_{1}(k)>0$. In the first case, when $\left|\varepsilon_{1}(k)\right|<\xi_{s}$, one of the nontrivial Dirac cones undergoes another gap-close-and-reopen process and becomes a trivial one with positive mass $\varepsilon_{1}(k)+\xi_{s}>0$. At each $\mathrm{M}$ point, only one nontrivial Dirac cone survives. The band evolution for this process is shown in Figs. 4(a1)-4(d2). In the second case, when $\left|\varepsilon_{1}(k)\right|<\xi_{s}$, one of the trivial Dirac cones undergoes a gap-close-and-reopen process and becomes a nontrivial one with negative mass $\varepsilon_{1}(k)-\xi_{s}<0$. At each $\mathrm{M}$ point, only one nontrivial Dirac cone emerges. The band evolution for this process is shown in Figs. 4(e1)-4(h2). In the first case, one needs relatively large SOC and the

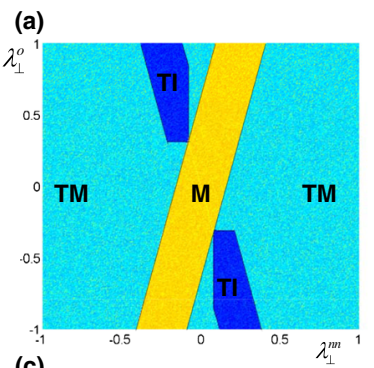

(c)
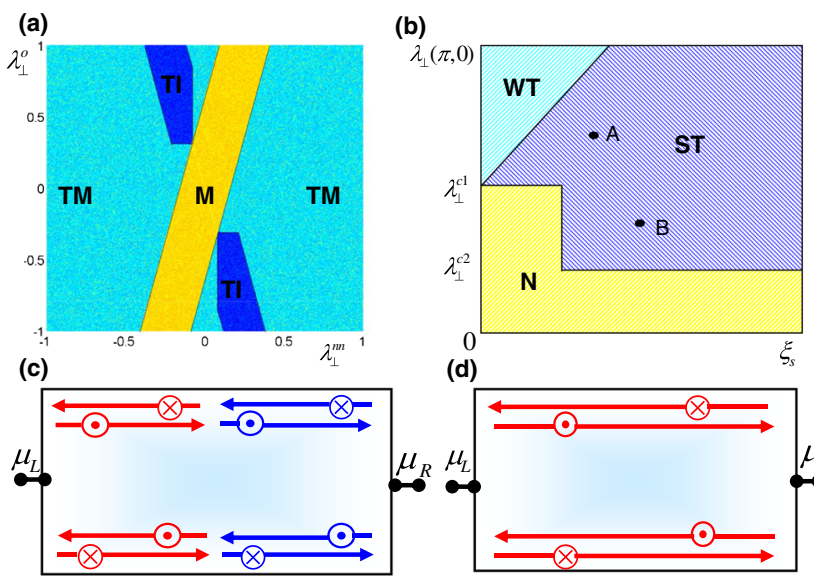

(d)
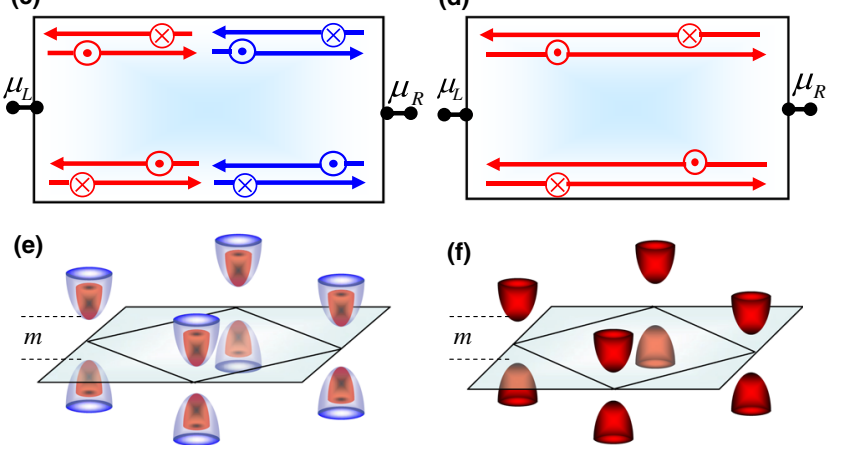

FIG. 5. (a) The phase diagram as a function of $\lambda_{\perp}^{o}$ and $\lambda_{\perp}^{n n}$. Here, M, TM, and TI label metallic, topologically metallic, and topologically insulating phases, respectively. (b) The phase diagram as a function of $\lambda_{\perp}(\pi, 0)$ and $\xi_{S}$ for $\lambda_{\perp}(\pi, 0)>0$. Here, WI, ST, and N label weak topological, strong topological, and normally trivial phases, respectively. "(c),(d)" The picture of the accumulation of spin currents in the weak and strong topological insulating phases of SL FeSe with the rectangle geometry. Here,

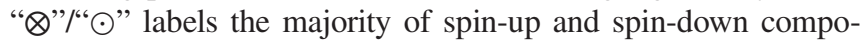
nents. The color corresponds to Fig. 3(i) and Fig. 4(d2) and 4(h2). (e) The two nontrivial (trivial) Dirac cones with negative (positive) mass $m$ correspond to Fig. 4(a1) [Fig. 4(e1)] around M points in BZ. Red and blue label the Dirac cone structures with even and odd parities, respectively. (f) The single nontrivial Dirac cone with negative mass $m$ [Figs. 4(c1) and 4(g1)] around M points in BZ. parity-broken coupling to overcome the trivial band gap and eliminate one nontrivial Dirac cone. In the second case, we can see that a finite value $H_{s}$ can create a region of a strong topological phase and can dramatically reduce the critical SOC that is necessary for a topological phase.

The phase diagrams for the topological transition are plotted in Fig. 5. In Fig. 5(a), we plot the phase diagram for the case of the weak topological phase with respect to the parameters $\lambda_{\perp}^{o}$ and $\lambda_{\perp}^{n n}$. Here, the topologically metal phase means the holelike band top at the $\Gamma$ point is higher than the electronlike band bottom around the $\mathrm{M}$ point so that we cannot tune the system into a full insulating phase; however, there is a gap at the $\mathrm{M}$ point to protect the topological phase. In the topologically insulating phase, the edge states propagate along the edges of the materials with opposite velocities for different spin components. The pictures of the edge states and the Dirac cones are schematically shown in Figs. 5(c) and 5(e), respectively. In Fig. 5(b), we plot the phase diagram for the $\lambda_{\perp}(k)>0$ case with respect to the parameter $\xi_{s}$ in $H_{s}$ and $\lambda_{\perp}(\pi, 0)$. The $\mathrm{A}$ and $\mathrm{B}$ points correspond to the cases shown in Figs. 4(c1) and 4(g1). The transport picture for the edge states is shown in Fig. 5(d), which matches the single nontrivial Dirac cone structure shown in Fig. 5(f).

\section{DISCUSSION AND SUMMARY}

There were several calculations on the electronic structures of the monolayer FeSe on $\mathrm{SrTiO}_{3}$ based on the DFT [30-32]. These works focus on the magnetic orders of the materials. In Ref. [30], the FeSe monolayer was argued to behave like a slightly doped semiconductor with a collinear antiferromagnetic order on the Fe lattice, and no strong hybridization of the electronic states between the substrate and the FeSe film was found. In Refs. [31,32], the checkerboard antiferromagnetic order on the Fe lattice was argued to be favored, and the hybridization between the substrate and the FeSe film was argued to be strong enough to suppress the hole pockets around the $\Gamma$ point. The existence of the magnetic order is still up for debate since experimentally it is extremely difficult to measure any magnetic order in the monolayer.

However, the band structure obtained from the DFT calculations in the normal state does not capture the essential gap opening at the $\mathrm{M}$ points, which is the key focus in this paper. In other words, the DFT calculation fails to capture the experimental results. In fact, it is understandable why it is difficult for DFT calculations to capture the gap behavior at the $\mathrm{M}$ point. If we compare the experiments with the DFT calculations, we find that all the DFT calculations assume the ideal interface connection between the monolayer FeSe and the substrate $\mathrm{SrTiO}_{3}$. Experimentally, the strong electron doping in the SL FeSe is realized by the annealing process, which may lead to loss of $\mathrm{Se}$ or $\mathrm{O}$ or rearrangement of $\mathrm{Fe}$ and $\mathrm{Se}$ on the $\mathrm{SrTiO}_{3}$ surface [5]. These potential effects may enhance the strain effect and strongly affect the 
orbital-dependent hoppings. Another point is that the correlation effect may play a role as well. It can adjust the Fermi level of the band of monolayer FeSe. In principle, this kind of correlation effect on band structure can also be renormalized into some hopping parameters. Capturing these complicated processes is beyond the current DFT capability. This is also the essential reason that motivates us to use the analytic approach combined with symmetry analysis to understand the gap behavior at the $\mathrm{M}$ point.

According to the aforementioned discussion, we see that the topological phase is associated with three key parameters: the trivial band gap, the intrinsic spin-orbital coupling strength at the M points, and the parity-broken coupling induced by the substrates. The bare spin-orbital coupling strength of Fe atoms is estimated to be around $80 \mathrm{meV}$ in Ref. [33] and $50 \mathrm{meV}$ in Ref. [34]. It can cause a bare energy splitting of about 50-100 meV around the $\mathrm{M}$ point. Considering the renormalization effect (about 4 in FeSe), the real splitting of the band caused by the SOC would be around $12-25 \mathrm{meV}$. Although we may replace Fe by heavier atoms such as $\mathrm{Ru}$ to further increase the SOC strength, the strength of the SOC, more or less, is a fixed quantity in the FeSe. However, the trivial band gap and parity-broken coupling at the M point can be engineered. With different substrates, the in-plane lattice constant can be tuned from 3.67 to $4 \AA$, which has recently been demonstrated in $\mathrm{FeSe} / \mathrm{STO} / \mathrm{KTO}[35]$ and FeSe/BTO [8] structures. There are many substrates that can be used for this interface engineering [8]. The trivial band gap can be estimated by the DFT calculations. For the isolated SL FeSe, when the lattice distortion of Fe-Fe bond changes from $3.95 \AA$, $3.97 \AA$ to $4.00 \AA$, the corresponding trivial band gap changes from $5 \mathrm{meV}, 18 \mathrm{meV}$ to $50 \mathrm{meV}$. If we assume the trivial band gap is $5 \mathrm{meV}$ in the single-layer FeSe, the weak topological phase needs $\lambda_{\perp}^{c 1}>5 \mathrm{meV}$ for $\xi_{s}=0$, and the strong topological phase needs $\lambda_{\perp}^{c 2}>2 \mathrm{meV}$ for $\xi_{s}>3 \mathrm{meV}$. Thus, the SOC of around $12-25 \mathrm{meV}$ is enough to realize the topological phase for SL FeSe with a lattice constant of about $3.95 \AA$. If the SL FeSe has a lattice constant of about $4.00 \AA ̊$, we need a quite large parity-broken coupling $\xi_{s}$ to overcome the trivial band gap of about $50 \mathrm{meV}$ to realize the strong topological phase. In Sec. I, we have shown the qualitative relationship between the gap and lattice parameters $t_{x}^{11}$. An accurate quantitative prediction of the trivial band gap on different substrates is beyond the capability of any current numerical methods. Nevertheless, as we know, in one limit, the large band gap (about $50 \mathrm{meV}$, measured by ARPES [8]) is created in the SL FeSe with STO and BTO substrates, and in the other limit, it vanishes in the bulk FeSe; we have shown the tunable gaps in a SL FeSe for several different lattice constants, and we believe that tunability should be realized by the interfacial engineering technique. Furthermore, the parity-broken coupling can be larger when the interaction with a substrate is stronger. $\xi_{s}$ is tunable with large flexibility. Hence, realizing the topological phase in the SL FeSe is very promising.

It is also possible to further extend the above analysis to the bulk materials. According to our above discussions, the difference of the electronic structures between the SL FeSe and the bulk FeSe mainly originates from the lattice distortion induced by the substrate, and the ratio between the height of $\mathrm{Se}$ and the Fe plane and the length of the Fe-Fe bond uniquely measures this difference. When the ratio declines less than the threshold value, a band gap is opened around the M point. Experimentally, this ratio can be tuned by applying internal or external pressure to the materials. Therefore, we suggest that this topological transition may be realized in the bulk materials of iron-based superconductors if the intensity of SOC is comparable to the band gap around the M point.

We want to emphasize advantages to realize the topological phase in iron-based superconductors. We noted that, recently, a topological insulator based on the BCS-type superconductor $\mathrm{BaBiO}_{3}$ was proposed, and a p-n junction fabricated with hole-doped and electron-doped $\mathrm{BaBiO}_{3}$ was argued to host Majorana fermions [36]; many conventional hybridized systems are proposed to realize topological superconductors [2] and Majorana fermions [37,38] in which the superconductivity is induced through the proximity effect of a conventional $s$-wave superconductor. Here, the superconducting mechanism in FeSe is believed to be beyond the BCS type, and the superconductivity can take place at much higher temperatures as the superconductivity behavior has been observed above $60 \mathrm{~K}$ in the single FeSe layer [5-7]. Furthermore, the superconductivity and topology is naturally integrated in the SL FeSe, and one may change doping and use different substrates to adjust such an integration. More interestingly, the SL FeSe system could be a topological superconductor at low temperatures. A time-reversal-invariant topological superconductor must satisfy two criterions [39]: (1) The system must have odd-parity pairing symmetry with a full superconducting gap, and (2) its Fermi surface must enclose an odd number of time-reversal-invariant momenta. The recently proposed odd-parity pairing scenario $[20,40]$ and the unique features of the electronic structures of SL FeSe can fulfill these two criterions. Detailed discussions are beyond the scope of the present paper, and we will present the results elsewhere.

Before we summarize the main results of this paper, we now discuss the experimental identification of the topological phase in the SL FeSe. Experimentally, there is a series of electrical and magnetic transport measurements that can be used to detect the basic signatures of the topological phase, and such kinds of measurements have been successfully adopted to verify the topological phase with a quantum-spin Hall effect in the famous two-dimensional (2D) HgTe-CdTe quantum-well system $[25,41]$. More relevantly, the SL FeSe system is comparable to the $\mathrm{HgTe}-\mathrm{CdTe}$ quantum-well system because the topological phase transition in the SL FeSe system is tuned by the strain effect while the topological 
phase transition in $\mathrm{HgTe}-\mathrm{CdTe}$ quantum wells is tuned by the thickness of the HgTe quantum well. Both the strain effect and the thickness of the quantum well can be easily modulated by current material-engineering techniques. Thus, the transport measurements on the HgTe-CdTe quantum wells can be utilized to detect the topological phase of the SL FeSe. The experimental signatures identifying the topologically trivial and nontrivial phases must meet the following criterions: (1) There should (not) exist a residual conductance plateau in the nontrivial (trivial) insulating regime as one varies the external gate voltage that is applied to tune the Fermi level, and the plateau should be independent of the sample width in the nontrivial phase, (2) the Hall conductance will present different values when one changes the gate voltage and external magnetic field in the nontrivial phase, but the Hall conductance vanishes in the trivial phase, and (3) at the edge of the sample, there should be spin accumulation that can be detected by spin-filtered measurements. It is also possible to measure edge states by scanning tunneling microscopy (STM) measurements. Recently, 1D topological edge states in Bi bilayer island structures formed on the surface of several different substrates (such as the clean $\mathrm{Bi}_{2} \mathrm{Te}_{3}$ and $\mathrm{Bi}(111)$-covered $\mathrm{Bi}_{2} \mathrm{Te}_{3}$, cleaved $\mathrm{Bi}_{2} \mathrm{Te}_{2} \mathrm{Se}$ crystal, and single Bi crystal [42-44]) have been identified by STM. In these experiments, the STM images clearly showed the existence of the edge states along the step edge of the $\mathrm{Bi}$ bilayer. Because of the structure analogy between the $\mathrm{Bi}$ bilayer grown on single $\mathrm{Bi}$ crystal and/or $\mathrm{Bi}_{2} \mathrm{Te}_{2}$ and $\mathrm{SL}$ FeSe grown on STO/KTO, similar STM measurements can also be carried out to identify the topological signatures in the $\mathrm{SL}$ FeSe system. Moreover, we expect that the identification should be easier in the SL FeSe system compared with the Bi bilayer system because the substrate STO/KTO in the SL FeSe system is a nonpolar pseudocubic band insulator with an electronic gap about $3.2 \mathrm{eV}$ for STO, while the substrates in the Bi bilayer system would have surface states that would probably hybridize with the edge states.

In conclusion, we show that the single-layer FeSe presents distinct and abundant structures compared with the bulk FeSe through its interaction with the substrates. We predict that there exists a strong topological phase in the SL FeSe. Thus, the single-layer FeSe not only serves as the building block for the iron-chalcogenide high-temperature superconductors, but it can also be an inherent topological material without doping. It is conceivable that many important physics applications can emerge when nontrivial topology and the high- $T_{c}$ superconductivity are intertwined within a single material.

\section{ACKNOWLEDGMENTS}

This work is supported by the Ministry of Science and Technology of China 973 program (No. 2012CB821400), the National Science Foundation of China (Grants No. 11334012, No. 91221303, and No. 11190020) and the "Strategic Priority Research Program (B)" of the Chinese Academy of Sciences,
Grant No. XDB07020200. We also thank King Abdulaziz University for financial support.

\section{APPENDIX A: TIGHT-BINDING HAMILTONIAN FOR BULK FeSe}

In lattice space, the tight-binding (TB) Hamiltonian is given by

$$
H_{t}=\sum_{\sigma} \sum_{m n} \sum_{i j}\left(t_{i j}^{m n}+\epsilon_{m} \delta_{m n} \delta_{i j}\right) d_{m, \sigma}^{\dagger}(i) d_{n, \sigma}(j) .
$$

Here, $\sigma$ labels the spin; $m, n$ label five $d$ orbitals; $i, j$ label the lattice site; $t_{i j}^{m n}$ are the corresponding hopping parameters; and $\epsilon_{m}$ is the on-site energy of the $d$ orbital. $d_{m, \sigma}^{\dagger}(i)$ creates a spin- $\sigma$ electron in the $m$ th orbital of $\mathrm{Fe}$ at site $i$. For convenience, we utilize $(1,2,3,4,5)$ to denote five $\left(x z, y z, x^{2}-y^{2}, x y, z^{2}\right)$ orbitals. In momentum space, the TB Hamiltonian is given by

$$
H_{t}=\sum_{k \sigma} \psi_{\sigma}^{\dagger}(q) H_{t}(q) \psi_{\sigma}(q) .
$$

Here, $\psi_{\sigma}(q)=\left[\left\{\psi_{A, \sigma}(q)\right\},\left\{\psi_{B, \sigma}(q)\right\}\right]^{T}$, with $\psi_{A / B, \sigma}(q)=$ $\left[d_{A / B, 1, \sigma}(q), d_{A / B, 2, \sigma}(q), d_{A / B, 3, \sigma}(q), d_{A / B, 4, \sigma}(q), d_{A / B, 5, \sigma}(q)\right]$ and $q=\left(q_{x}, q_{y}\right)$, and it is defined in two-Fe BZ. The dimension of $H_{t}(q)$ is $10 \times 10$ because of the two-Fe unit cell. Theoretically, the effective one-Fe unit-cell picture is usually used for simplicity. The two-Fe unit-cell picture and the one-Fe unit-cell picture are connected through a unitary transformation. It is easy to check that

$$
U H_{t}(q) U^{\dagger}=\left[\begin{array}{cc}
A(k) & 0 \\
0 & A\left(k^{\prime}\right)
\end{array}\right] .
$$

Here, $k^{\prime}=k+Q$ with $Q=(\pi, \pi)$, and $k$ is defined in one-Fe BZ.

$$
U=\left[\begin{array}{cc}
U_{A} & U_{B} \\
U_{A} & -U_{B}
\end{array}\right]
$$

with

$$
\begin{aligned}
& U_{A}=\frac{\sqrt{2}}{2}\left[\begin{array}{lllll}
1 & 0 & 0 & 0 & 0 \\
0 & 1 & 0 & 0 & 0 \\
0 & 0 & 1 & 0 & 0 \\
0 & 0 & 0 & 1 & 0 \\
0 & 0 & 0 & 0 & 1
\end{array}\right], \\
& U_{B}=\frac{\sqrt{2}}{2}\left[\begin{array}{ccccc}
1 & 0 & 0 & 0 & 0 \\
0 & 1 & 0 & 0 & 0 \\
0 & 0 & -1 & 0 & 0 \\
0 & 0 & 0 & -1 & 0 \\
0 & 0 & 0 & 0 & -1
\end{array}\right] .
\end{aligned}
$$


$A(k)$ is the general one-Fe unit-cell TB Hamiltonian in the basis $\phi_{\sigma}(k)=\left[d_{1, \sigma}(k), d_{2, \sigma}(k), d_{3, \sigma}(k), d_{4, \sigma}(k), d_{5, \sigma}(k)\right]^{T}$. The connection between the two bases is

$$
\begin{aligned}
d_{1 / 2, \sigma}(k) & =\frac{\sqrt{2}}{2}\left[d_{A, 1 / 2, \sigma}(q)+d_{B, 1 / 2, \sigma}(q)\right] \\
d_{1 / 2, \sigma}\left(k^{\prime}\right) & =\frac{\sqrt{2}}{2}\left[d_{A, 1 / 2, \sigma}(q)-d_{B, 1 / 2, \sigma}(q)\right] \\
d_{3 / 4 / 5, \sigma}(k) & =\frac{\sqrt{2}}{2}\left[d_{A, 3 / 4 / 5, \sigma}(q)-d_{B, 3 / 4 / 5, \sigma}(q)\right] \\
d_{3 / 4 / 5, \sigma}\left(k^{\prime}\right) & =\frac{\sqrt{2}}{2}\left[d_{A, 3 / 4 / 5, \sigma}(q)+d_{B, 3 / 4 / 5, \sigma}(q)\right] .
\end{aligned}
$$

The nonzero terms in $A(k)$ are listed as follows:

$$
\begin{aligned}
A_{11}(k)= & \epsilon_{1}+2 t_{x}^{11} \cos k_{x}+2 t_{y}^{11} \cos k_{y}+4 t_{x y}^{11} \cos k_{x} \cos k_{y} \\
& +2 t_{x x}^{11} \cos 2 k_{x}+2 t_{y y}^{11} \cos 2 k_{y}+4 t_{x x y}^{11} \cos 2 k_{x} \cos k_{y} \\
& +4 t_{x y y}^{11} \cos k_{x} \cos 2 k_{y}+4 t_{x x y y}^{11} \cos 2 k_{x} \cos 2 k_{y} \\
A_{22}(k)= & \epsilon_{1}+2 t_{y}^{11} \cos k_{x}+2 t_{x}^{11} \cos k_{y}+4 t_{x y}^{11} \cos k_{x} \cos k_{y} \\
& +2 t_{y y}^{11} \cos 2 k_{x}+2 t_{x x}^{11} \cos 2 k_{y}+4 t_{y y x}^{11} \cos 2 k_{x} \cos k_{y} \\
& +4 t_{x x y}^{11} \cos k_{x} \cos 2 k_{y}+4 t_{x x y y}^{11} \cos 2 k_{x} \cos 2 k_{y} \\
A_{33}(k)= & \epsilon_{3}+2 t_{x}^{33}\left(\cos k_{x}+\cos k_{y}\right)+4 t_{x y}^{33} \cos k_{x} \cos k_{y} \\
A_{44}(k)= & \epsilon_{4}+2 t_{x}^{44}\left(\cos k_{x}+\cos k_{y}\right)+4 t_{x y}^{44} \cos k_{x} \cos k_{y} \\
& +4 t_{x x y}^{44}\left(\cos 2 k_{x} \cos k_{y}+\cos k_{x} \cos 2 k_{y}\right) \\
& +4 t_{x x y y}^{44} \cos 2 k_{x} \cos 2 k_{y} \\
A_{55}(k)= & \epsilon_{5} \\
A_{12}(k)= & -4 t_{x y}^{12} \sin k_{x} \sin k_{y} \\
A_{13 / 23}(k)= & \pm 2 i t_{x}^{13} \sin k_{y / x} \pm 4 i t_{x y}^{13} \sin k_{y / x} \cos k_{x / y} \\
A_{14 / 24}(k)= & -2 i t_{x}^{14} \sin k_{x / y}+4 i t_{x y}^{14} \sin k_{x / y} \cos k_{y / x} \\
A_{15 / 25}(k)= & 2 i t_{x}^{15} \sin k_{y / x}+4 i t_{x y}^{15} \sin k_{y / x} \cos k_{x / y} \\
A_{35}(k)= & 2 t_{x}^{35}\left(\cos k_{x}-\cos k_{y}\right) \\
A_{45}(k)= & -4 t_{x y}^{45} \sin k_{x} \sin k_{y} .
\end{aligned}
$$

TABLE I. Intra-orbital hopping parameters

\begin{tabular}{lcccc}
\hline \hline$t_{i}^{m n}$ & $m n=11$ & $m n=33$ & $m n=44$ & $m n=55$ \\
\hline$i=x$ & -0.08 & 0.412 & 0.063 & 0 \\
$i=y$ & -0.311 & 0 & 0 & 0 \\
$i=x y$ & 0.232 & -0.066 & 0.086 & 0 \\
$i=x x$ & 0.009 & 0 & 0 & 0 \\
$i=y y$ & -0.045 & 0 & 0 & 0 \\
$i=x x y$ & -0.016 & 0 & -0.017 & 0 \\
$i=x y y$ & 0.019 & 0 & 0 & 0 \\
$i=x x y y$ & 0.02 & 0 & -0.028 & 0 \\
\hline \hline
\end{tabular}

TABLE II. Interorbital hopping parameters

\begin{tabular}{lcccccc}
\hline \hline$t_{i}^{m n}$ & $m n=12$ & $m n=13$ & $m n=14$ & $m n=14$ & $m n=35$ & $m n=45$ \\
\hline$i=x$ & 0 & 0.3 & 0.305 & -0.18 & 0.338 & 0 \\
$i=x y$ & 0.099 & -0.089 & -0.056 & 0.146 & 0 & -0.109 \\
\hline \hline
\end{tabular}

The on-site orbital energy is $\epsilon_{1}=\epsilon_{2}=0.02, \epsilon_{3}=$ $-0.539, \epsilon_{4}=0.014, \epsilon_{5}=-0.581$. The hopping parameters are shown in Tables I and II [45].

\section{APPENDIX B: THE INFLUENCE OF LATTICE DISTORTION TO A HOPPING PARAMETER}

In this section, we present a detailed discussion about the influence of the lattice distortion to the hopping parameters. The overlap integrals between different orbitals can be calculated with the Slater-Koster method [22]. In ironbased materials, the band structures around the Fermi surface mainly have $d_{x z}, d_{y z}$, and $d_{x y}$ characters. Furthermore, we can find that the difference of band structure between the bulk and single-layer cases is mainly affected by the change of the hoppings between these three orbitals. Now, we focus on the three orbitals.

Consider the Fe-Se trilayer structure shown in Fig. 6(a). The direction cosines for two centers with coordinates $\mathbf{R}_{1}\left(x_{1}, y_{1}, z_{1}\right)$ and $\mathbf{R}_{2}\left(x_{2}, y_{2}, z_{2}\right)$ are defined as $l=$ $\cos \theta_{x}=\left(x_{2}-x_{1}\right) /\left|\mathbf{R}_{2}-\mathbf{R}_{1}\right|, \quad m=\cos \theta_{y}=\left(y_{2}-y_{1}\right) /$ $\left|\mathbf{R}_{2}-\mathbf{R}_{1}\right|$, and $n=\cos \theta_{z}=\left(z_{2}-z_{1}\right) /\left|\mathbf{R}_{2}-\mathbf{R}_{1}\right|$. The overlap integrals are listed in Table III.

The effective Fe-Fe hoppings include two parts: the direct hoppings from the direct overlap integrals of $d$ orbitals and the indirect hoppings through the bridge of $p$ orbitals. In order to consider the indirect $\mathrm{Fe}-\mathrm{Fe}$ hoppings through the $p$ orbitals, we must first consider the Fe-Se hoppings. Define the ratio $x=h / k$, and then the Fe-Se hoppings can be parametrized as
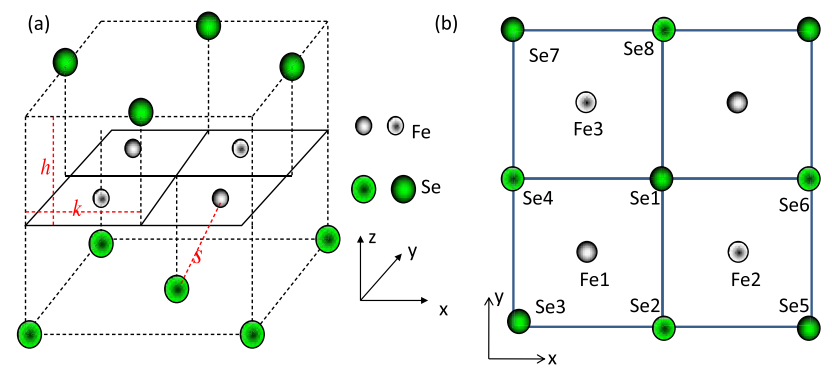

FIG. 6. The lattice structure of the FeSe trilayer is shown in (a). $k$ is the nearest-neighbor Fe-Fe distance. $h$ is the distance between $\mathrm{Se}$ and Fe layers. $s$ is the nearest-neighbor $\mathrm{Fe}-\mathrm{Se}$ distance. Panel (b) is the top view of (a), and the Fe and Se atoms are numbered. 
TABLE III. Overlap integrals for $p$ and $d$ orbitals.

\begin{tabular}{lc}
\hline \hline$E_{x, y z}$ & $\sqrt{3} \operatorname{lm} n(p d \sigma)-2 \operatorname{lmn}(p d \pi)$ \\
$E_{x, x z}$ & $\sqrt{3} l^{2} n(p d \sigma)-n\left(1-2 l^{2}\right)(p d \pi)$ \\
$E_{y, y z}$ & $\sqrt{3} m^{2} n(p d \sigma)-n\left(1-2 m^{2}\right)(p d \pi)$ \\
$E_{x, x y}$ & $\sqrt{3} l^{2} m(p d \sigma)+m\left(1-2 l^{2}\right)(p d \pi)$ \\
$E_{z, x z}$ & $\sqrt{3} n^{2} l(p d \sigma)+l\left(1-2 n^{2}\right)(p d \pi)$ \\
$E_{z, y z}$ & $\sqrt{3} n^{2} m(p d \sigma)+m\left(1-2 n^{2}\right)(p d \pi)$ \\
$E_{z, x y}$ & $\sqrt{3} \operatorname{lm} n(p d \sigma)-2 \operatorname{lm} n(p d \pi)$ \\
$E_{x z-x z}$ & $3 l^{2} n^{2}(d d \sigma)+\left(l^{2}+n^{2}-4 l^{2} n^{2}\right)(d d \pi)$ \\
& $+\left(m^{2}+l^{2} n^{2}\right)(d d \delta)$ \\
$E_{x z-y z}$ & $3 \operatorname{lm}^{2}(d d \sigma)+l m\left(1-4 n^{2}\right)[(d d \pi)-(d d \delta)]$ \\
$E_{y z-y z}$ & $3 m^{2} n^{2}(d d \sigma)+\left(m^{2}+n^{2}-4 m^{2} n^{2}\right)(d d \pi)$ \\
& $+\left(l^{2}+m^{2} n^{2}\right)(d d \delta)$ \\
$E_{x y-x y}$ & $3 l^{2} m^{2}(d d \sigma)+\left(l^{2}+m^{2}-4 l^{2} m^{2}\right)(d d \pi)$ \\
& $+\left(n^{2}+l^{2} m^{2}\right)(d d \delta)$ \\
\hline \hline
\end{tabular}

$$
\begin{aligned}
&\left|t_{x, x z}\right|=\mid\left|t_{y, y z}\right|=\frac{\sqrt{3}}{4} x f^{3}(x) E_{(p d \sigma)}[s(x)] \\
&+ {\left[x f(x)-\frac{1}{2} x f^{3}(x)\right] E_{(p d \pi)}[s(x)] } \\
&\left|t_{x, y z}\right|=\left|t_{y, x z}\right|=\frac{\sqrt{3}}{4} x f^{3}(x) E_{(p d \sigma)}[s(x)] \\
&-\frac{1}{2} x f^{3}(x) E_{(p d \pi)}[s(x)] \\
&\left|t_{x / y, x y}\right|= \frac{\sqrt{3}}{8} f^{3}(x) E_{(p d \sigma)}[s(x)] \\
&+\left[\frac{1}{2} f(x)-\frac{1}{4} f^{3}(x)\right] E_{(p d \pi)}[s(x)] \\
&\left|t_{z, x z / y z}\right|= \frac{\sqrt{3}}{2} x^{2} f^{3}(x) E_{(p d \sigma)}[s(x)] \\
&+\left[f(x)-2 x^{2} f^{3}(x)\right] E_{(p d \pi)}[s(x)] \\
&\left|t_{z, x y}\right|=\frac{\mathrm{B}}{2} x^{2} f^{3}(x) E_{(p d \sigma)}[s(x)]-x^{2} f^{3}(x) E_{(p d \pi)}[s(x)]
\end{aligned}
$$

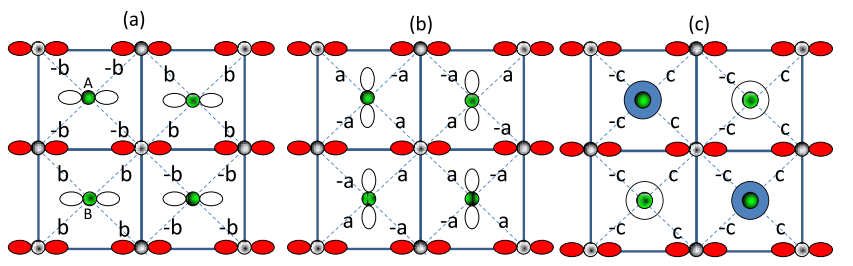

FIG. 7. The pattern of $d_{x z}-p$ couplings. Here, $a=\left|t_{y, x z}\right|$, $b=\left|t_{x, x z}\right|, c=\left|t_{z, x z}\right|$.
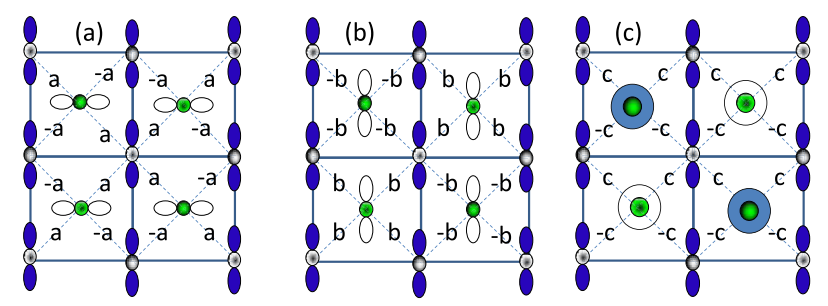

FIG. 8. The pattern of $d_{y z}-p$ couplings. Here, $a=\left|t_{y, x z}\right|$, $b=\left|t_{x, x z}\right|, c=\left|t_{z, x z}\right|$.

Here,

$$
f(x)=\frac{1}{\sqrt{\frac{1}{2}+x^{2}}}
$$

and $s(x)=k \sqrt{(1 / 2)+x^{2}}$ is the nearest Fe-Se distance; we use $E_{(p d \sigma / \pi)}[s(x)]$ to denote the energy of $p d \sigma / \pi$ bonds. The direct Fe-Fe hoppings are straightforward, and both the nearest and next-nearest ones have three types, namely, $E_{(d d \sigma / \pi / \delta)}(k)$ and $E_{(d d \sigma / \pi / \delta)}(\sqrt{2} k)$.

$$
\begin{gathered}
\left|t_{x z, x z}^{(x)}\right|=\left|t_{y z, y z}^{(y)}\right|=E_{(d d \pi)}[k] \\
\left|t_{x z, x z}^{(y)}\right|=\left|t_{y z, y z}^{(x)}\right|=E_{(d d \delta)}[k] \\
\left|t_{x y, x y}\right|=E_{(d d \pi)}[k] \\
\left|t_{x z, x z}^{\prime}\right|=\left|t_{y z, y z}^{\prime}\right|=E_{(d d \pi)}[\sqrt{2} k]+E_{(d d \delta)}[\sqrt{2} k] \\
\left|t_{x y, x y}^{\prime}\right|=3 E_{(d d \sigma)}[\sqrt{2} k]-2 E_{(d d \pi)}[\sqrt{2} k]+E_{(d d \delta)}[\sqrt{2} k] \\
\left|t_{x z, y z}^{\prime}\right|=E_{(d d \pi)}[\sqrt{2} k]-E_{(d d \delta)}[\sqrt{2} k]
\end{gathered}
$$

In order to calculate the effective Fe-Fe hoppings, we consider a Fe-Se cluster that involves nine Se and four Fe; it is shown in Fig. 6(b). The coupling patterns between $d$ orbitals and $p$ orbitals are shown in Figs. 7-9.

According to Figs. 7-9, the new orthonormal wave function for the local $d$ orbitals can be constructed as follows:
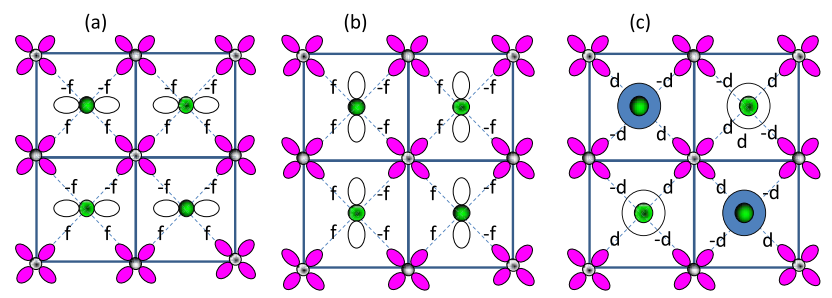

FIG. 9. The pattern of $d_{x y}-p$ couplings. Here, $f=\left|t_{x, x y}\right|$. 


$$
\begin{aligned}
\left|d_{1, x z}\right\rangle^{\prime}= & \frac{1}{\sqrt{1+4 \alpha_{1}^{2}+4 \beta_{1}^{2}+4 \gamma_{1}^{2}}} \times\left\{\left|d_{1, x z}\right\rangle-\alpha_{1}\left|p_{1, x}-p_{2, x}\right\rangle-\beta_{1}\left|p_{1, y}+p_{2, y}\right\rangle-\gamma_{1}\left|p_{1, z}+p_{2, z}\right\rangle\right. \\
& \left.-\alpha_{1}\left|p_{3, x}-p_{4, x}\right\rangle-\beta_{1}\left|p_{3, y}+p_{4, y}\right\rangle+\gamma_{1}\left|p_{3, z}+p_{4, z}\right\rangle\right\} \\
= & \frac{1}{\sqrt{1+4 \alpha_{1}^{2}+4 \beta_{1}^{2}+4 \gamma_{1}^{2}}} \times\left\{\left|d_{1, x z}\right\rangle-\alpha_{1}\left|p_{1, x}-p_{4, x}\right\rangle-\beta_{1}\left|p_{1, y}+p_{4, y}\right\rangle-\gamma_{1}\left|p_{1, z}-p_{4, z}\right\rangle\right. \\
& \left.-\alpha_{1}\left|p_{3, x}-p_{2, x}\right\rangle-\beta_{1}\left|p_{3, y}+p_{2, y}\right\rangle+\gamma_{1}\left|p_{3, z}-p_{2, z}\right\rangle\right\},
\end{aligned}
$$

$$
\begin{aligned}
\left|d_{2, x z}\right\rangle^{\prime}= & \frac{1}{\sqrt{1+4 \alpha_{1}^{2}+4 \beta_{1}^{2}+4 \gamma_{1}^{2}}} \times\left\{\left|d_{2, x z}\right\rangle-\alpha_{1}\left|p_{1, x}-p_{2, x}\right\rangle+\beta_{1}\left|p_{1, y}+p_{2, y}\right\rangle+\gamma_{1}\left|p_{1, z}+p_{2, z}\right\rangle\right. \\
& \left.-\alpha_{1}\left|p_{5, x}-p_{6, x}\right\rangle+\beta_{1}\left|p_{5, y}+p_{6, y}\right\rangle-\gamma_{1}\left|p_{5, z}+p_{6, z}\right\rangle\right\},
\end{aligned}
$$

$$
\begin{aligned}
\left|d_{3, x z}\right\rangle^{\prime}= & \frac{1}{\sqrt{1+4 \alpha_{1}^{2}+4 \beta_{1}^{2}+4 \gamma_{1}^{2}}} \times\left\{\left|d_{3, x z}\right\rangle-\alpha_{1}\left|p_{1, x}-p_{4, x}\right\rangle+\beta_{1}\left|p_{1, y}+p_{4, y}\right\rangle-\gamma_{1}\left|p_{1, z}-p_{4, z}\right\rangle\right. \\
& \left.-\alpha_{1}\left|p_{7, x}-p_{8, x}\right\rangle+\beta_{1}\left|p_{7, y}+p_{8, y}\right\rangle+\gamma_{1}\left|p_{7, z}-p_{8, z}\right\rangle\right\},
\end{aligned}
$$

$$
\begin{aligned}
\left|d_{1, y z}\right\rangle^{\prime}= & \frac{1}{\sqrt{1+4 \alpha_{1}^{2}+4 \beta_{1}^{2}+4 \gamma_{1}^{2}}} \times\left\{\left|d_{1, y z}\right\rangle-\beta_{1}\left|p_{1, x}+p_{2, x}\right\rangle-\alpha_{1}\left|p_{1, y}-p_{2, y}\right\rangle-\gamma_{1}\left|p_{1, z}-p_{2, z}\right\rangle\right. \\
& \left.-\beta_{1}\left|p_{3, x}+p_{4, x}\right\rangle-\alpha_{1}\left|p_{3, y}-p_{4, y}\right\rangle+\gamma_{1}\left|p_{3, z}-p_{4, z}\right\rangle\right\} \\
= & \frac{1}{\sqrt{1+4 \alpha_{1}^{2}+4 \beta_{1}^{2}+4 \gamma_{1}^{2}}} \times\left\{\left|d_{1, y z}\right\rangle-\beta_{1}\left|p_{1, x}+p_{4, x}\right\rangle-\alpha_{1}\left|p_{1, y}-p_{4, y}\right\rangle-\gamma_{1}\left|p_{1, z}+p_{4, z}\right\rangle\right. \\
& -\beta_{1}\left|p_{3, x}+p_{2, x}\right\rangle-\alpha_{1}\left|p_{3, y}-p_{2, y}\right\rangle+\gamma_{1}\left|p_{3, z}+p_{2, z}\right\rangle,
\end{aligned}
$$

$$
\begin{aligned}
\left|d_{2, y z}\right\rangle^{\prime}= & \frac{1}{\sqrt{1+4 \alpha_{1}^{2}+4 \beta_{1}^{2}+4 \gamma_{1}^{2}}} \times\left\{\left|d_{2, y z}\right\rangle+\beta_{1}\left|p_{1, x}+p_{2, x}\right\rangle-\alpha_{1}\left|p_{1, y}-p_{2, y}\right\rangle-\gamma_{1}\left|p_{1, z}-p_{2, z}\right\rangle\right. \\
& \left.+\beta_{1}\left|p_{5, x}+p_{6, x}\right\rangle-\alpha_{1}\left|p_{5, y}-p_{6, y}\right\rangle+\gamma_{1}\left|p_{5, z}-p_{6, z}\right\rangle\right\},
\end{aligned}
$$

$$
\left|d_{3, y z}\right\rangle^{\prime}=\frac{1}{\sqrt{1+4 \alpha_{1}^{2}+4 \beta_{1}^{2}+4 \gamma_{1}^{2}}} \times\left\{\left|d_{3, y z}\right\rangle+\beta_{1}\left|p_{1, x}+p_{4, x}\right\rangle-\alpha_{1}\left|p_{1, y}-p_{4, y}\right\rangle+\gamma_{1}\left|p_{1, z}+p_{4, z}\right\rangle\right.
$$$$
\left.+\beta_{1}\left|p_{7, x}+p_{8, x}\right\rangle-\alpha_{1}\left|p_{7, y}-p_{8, y}\right\rangle-\gamma_{1}\left|p_{7, z}-p_{8, z}\right\rangle\right\}
$$

$$
\begin{aligned}
\left|d_{1, x y}\right\rangle^{\prime}= & \frac{1}{\sqrt{1+8 \alpha_{4}^{2}+4 \gamma_{4}^{2}}} \times\left\{\left|d_{1, x y}\right\rangle+\alpha_{4}\left|p_{1, x}-p_{2, x}\right\rangle+\alpha_{4}\left|p_{1, y}+p_{2, y}\right\rangle-\gamma_{4}\left|p_{1, z}+p_{2, z}\right\rangle\right. \\
& \left.-\alpha_{4}\left|p_{3, x}-p_{4, x}\right\rangle-\alpha_{4}\left|p_{3, y}+p_{4, y}\right\rangle-\gamma_{4}\left|p_{3, z}+p_{4, z}\right\rangle\right\} \\
= & \frac{1}{\sqrt{1+8 \alpha_{4}^{2}+4 \gamma_{4}^{2}}} \times\left\{\left|d_{1, x y}\right\rangle+\alpha_{4}\left|p_{1, x}+p_{4, x}\right\rangle+\alpha_{4}\left|p_{1, y}-p_{4, y}\right\rangle-\gamma_{4}\left|p_{1, z}+p_{4, z}\right\rangle\right. \\
& \left.-\alpha_{4}\left|p_{3, x}+p_{2, x}\right\rangle-\alpha_{4}\left|p_{3, y}-p_{2, y}\right\rangle-\gamma_{4}\left|p_{3, z}+p_{2, z}\right\rangle\right\},
\end{aligned}
$$

$$
\begin{aligned}
\left|d_{2, x y}\right\rangle^{\prime}= & \frac{1}{\sqrt{1+8 \alpha_{4}^{2}+4 \gamma_{4}^{2}}} \times\left\{\left|d_{2, x y}\right\rangle+\alpha_{4}\left|p_{1, x}-p_{2, x}\right\rangle-\alpha_{4}\left|p_{1, y}+p_{2, y}\right\rangle+\gamma_{4}\left|p_{1, z}+p_{2, z}\right\rangle\right. \\
& \left.-\alpha_{4}\left|p_{5, x}-p_{6, x}\right\rangle+\alpha_{4}\left|p_{5, y}+p_{6, y}\right\rangle+\gamma_{4}\left|p_{5, z}+p_{6, z}\right\rangle\right\}
\end{aligned}
$$

$$
\begin{aligned}
\left|d_{3, x y}\right\rangle^{\prime}= & \frac{1}{\sqrt{1+8 \alpha_{4}^{2}+4 \gamma_{4}^{2}}} \times\left\{\left|d_{3, x y}\right\rangle+\alpha_{4}\left|p_{1, x}+p_{4, x}\right\rangle-\alpha_{4}\left|p_{1, y}-p_{4, y}\right\rangle+\gamma_{4}\left|p_{1, z}+p_{4, z}\right\rangle\right. \\
& \left.-\alpha_{4}\left|p_{7, x}+p_{8, x}\right\rangle+\alpha_{4}\left|p_{7, y}-p_{8, y}\right\rangle+\gamma_{4}\left|p_{7, z}+p_{8, z}\right\rangle\right\}
\end{aligned}
$$


where

$$
\begin{aligned}
& \alpha_{1}=\frac{\left\langle p_{1, x}-p_{2, x}|H| d_{1, x z}+d_{2, x z}\right\rangle}{4\left(\epsilon_{p_{x}}-\epsilon_{d_{x z}}\right)}=\left|\frac{t_{x, x z}}{\epsilon_{p_{x}}-\epsilon_{d_{x z}}}\right|, \\
& \beta_{1}=\frac{\left\langle p_{1, y}+p_{2, y}|H| d_{1, x z}-d_{1, x z}\right\rangle}{4\left(\epsilon_{p_{y}}-\epsilon_{d_{x z}}\right)}=\left|\frac{t_{y, x z}}{\epsilon_{p_{y}}-\epsilon_{d_{x z}}}\right|, \\
& \gamma_{1}=\frac{\left\langle p_{1, z}+p_{2, z}|H| d_{1, x z}-d_{2, x z}\right\rangle}{4\left(\epsilon_{p_{z}}-\epsilon_{d_{x z}}\right)}=\left|\frac{t_{z, x z}}{\epsilon_{p_{z}}-\epsilon_{d_{x z}}}\right|, \\
& \alpha_{4}=\frac{-\left\langle p_{1, x}-p_{2, x}|H| d_{1, x y}-d_{2, x y}\right\rangle}{4\left(\epsilon_{p_{x}}-\epsilon_{d x y}\right)}=\left|\frac{t_{x, x y}}{\epsilon_{p_{x}}-\epsilon_{d_{x y}}}\right|, \\
& \gamma_{4}=\frac{\left\langle p_{1, z}+p_{2, z}|H| d_{1, x z}-d_{2, x z}\right\rangle}{4\left(\epsilon_{p_{z}}-\epsilon_{d_{x y}}\right)}=\left|\frac{t_{z, x y}}{\epsilon_{p_{z}}-\epsilon_{d_{x y}}}\right| .
\end{aligned}
$$

Note that the $\alpha_{i}, \beta_{i}$, and $\gamma_{i}$ in Eqs. (B22)-(B26) are less than 1; we only keep the terms to the first order. The effective Fe-Fe hoppings can be obtained as follows.

The effective nearest-neighbor $d_{x z}-d_{x z}$ hopping along the $\vec{x}$ direction is

$$
\begin{aligned}
t_{x}^{11}= & \left\langle\left. d_{1, x z}\right|^{\prime} H \mid d_{2, x z}\right\rangle^{\prime} \\
\sim & \frac{1}{1+4 \alpha_{1}^{2}+4 \beta_{1}^{2}+4 \gamma_{1}^{2}} \\
& \times\left(t_{x z, x z}^{(x)}+4 \alpha_{1}\left|t_{x, x z}\right|-4 \beta_{1}\left|t_{y, x z}\right|+4 \gamma_{1}\left|t_{z, x z}\right|\right) .
\end{aligned}
$$

The effective nearest-neighbor $d_{x z}-d_{x z}$ hopping along the $\vec{y}$ direction is

$$
\begin{aligned}
t_{y}^{11}= & \left\langle\left. d_{1, x z}\right|^{\prime} H \mid d_{3, x z}\right\rangle^{\prime} \\
\sim & \frac{1}{1+4 \alpha_{1}^{2}+4 \beta_{1}^{2}+4 \gamma_{1}^{2}} \\
& \times\left(t_{x z, x z}^{(y)}+4 \alpha_{1}\left|t_{x, x z}\right|-4 \beta_{1}\left|t_{y, x z}\right|-4 \gamma_{1}\left|t_{z, x z}\right|\right) .
\end{aligned}
$$

The effective next-nearest-neighbor $d_{x z}-d_{x z}$ hopping is

$$
\begin{aligned}
t_{x y}^{11}= & \left\langle\left. d_{2, x z}\right|^{\prime} H \mid d_{3, x z}\right\rangle^{\prime} \\
\sim & \frac{1}{1+4 \alpha_{1}^{2}+4 \beta_{1}^{2}+4 \gamma_{1}^{2}} \\
& \times\left(t_{x z, x z}^{\prime}+2 \alpha_{1}\left|t_{x, x z}\right|+2 \beta_{1}\left|t_{y, x z}\right|-2 \gamma_{1}\left|t_{z, x z}\right|\right) .
\end{aligned}
$$

The effective nearest-neighbor $d_{x y}-d_{x y}$ hopping is

$$
\begin{aligned}
t_{x}^{44} & =\left\langle\left. d_{1, x y}\right|^{\prime} H \mid d_{2, x y}\right\rangle^{\prime} \\
& \sim \frac{1}{1+8 \alpha_{4}^{2}+8 \gamma_{4}^{2}}\left(-t_{x y, x y}-4 \gamma_{4}\left|t_{z, x y}\right|\right) .
\end{aligned}
$$

The effective next-nearest-neighbor $d_{x y}-d_{x y}$ hopping is

$$
\begin{aligned}
t_{x y}^{44} & =\left\langle\left. d_{2, x y}\right|^{\prime} H \mid d_{3, x y}\right\rangle^{\prime} \\
& \sim \frac{1}{1+8 \alpha_{4}^{2}+8 \gamma_{4}^{2}}\left(t_{x y, x y}^{\prime}-4 \alpha_{4}\left|t_{x, x y}\right|+2 \gamma_{4}\left|t_{z, x y}\right|\right) .
\end{aligned}
$$

The effective nearest-neighbor $d_{x z}-d_{x y}$ hopping along the $\vec{x}$ direction is

$$
\begin{aligned}
t_{x}^{14}= & \left\langle\left. d_{1, x z}\right|^{\prime} H \mid d_{2, x y}\right\rangle^{\prime} \\
\sim & \frac{1}{\sqrt{1+4 \alpha_{1}^{2}+4 \beta_{1}^{2}+4 \gamma_{1}^{2}}} \frac{1}{\sqrt{1+8 \alpha_{4}^{2}+4 \gamma_{4}^{2}}} \\
& \times\left[2\left(\beta_{1}-\alpha_{1}\right)\left|t_{x, x y}\right|-2 \gamma_{1}\left|t_{z, x y}\right|+2 \alpha_{4}\left(-\left|t_{x, x z}\right|+\left|t_{y, x z}\right|\right)\right. \\
& \left.-2 \gamma_{4}\left|t_{z, x z}\right|\right] .
\end{aligned}
$$

Equations (B27)-(B32) can be used to qualitatively calculate the hopping parameters, and we use them to study the changes of hoppings due to the lattice distortion. In order to calculate the above effective $d-d$ hoppings, we must know the energy of the $(p d \sigma / \pi)$ and $(d d \sigma / \pi / \delta)$ bonds. The generic forms of $E_{(p / d d \sigma / \pi / \delta)}[R]$ [46] can be approximatively expressed as

$E_{\left(\alpha \alpha^{\prime} \mu\right)}[R]=\left[a_{\alpha \alpha^{\prime} \mu}+b_{\alpha \alpha^{\prime} \mu} R+c_{\alpha \alpha^{\prime} \mu} R^{2}\right] \exp \left(-d_{\alpha \alpha^{\prime} \mu}^{2} R\right) F(R)$.

Here, $a_{\alpha \alpha^{\prime} \mu}, b_{\alpha \alpha^{\prime} \mu}, c_{\alpha \alpha^{\prime} \mu}$, and $d_{\alpha \alpha^{\prime} \mu}$ are fitting parameters; $\alpha$, $\alpha^{\prime}$ label $p$ or $d$ orbitals; $\mu$ labels $\sigma, \pi$, or $\delta$ bonds; $R$ is the distance between two atoms; $F(R)$ is the cutoff function; and $F(R)=1 /\left\{1+\exp \left[\left(R-R_{0}\right) / L_{0}\right]\right\}$ when $R<R_{0}$ and $F(R)=0$ when $R>R_{0}$. We set $R_{0}=14$ in Bohr units and $L_{0}=0.5$. Another point is that all the iron-based materials have the same $\mathrm{Fe}-\mathrm{Se}(\mathrm{As})$ trilayer structure except for the slightly difference of lattice constant. Hence, it is natural to assume that the effect of lattice distortion for different materials follows the same rule. Here, we take $\mathrm{LaOFeAs}$ as an example. In the bulk case, we set $k_{b}=5.329 \mathrm{Bohr}$ and $x_{b}=0.4838$. For the single-layer case, we set $k_{s}=5.6551$ in order to keep the same distortion ratio of $6.12 \%$ compared with the FeSe case. The on-site orbital energy is set as $\epsilon_{p_{x / y / z}}=0.18566, \quad \epsilon_{d_{x z / y z}}=0.51108, \quad \epsilon_{d_{x y}}=$ $0.54617, \epsilon_{d_{x^{2}-y^{2}}}=0.54548$, and $\epsilon_{d_{z^{2}}}=0.5513$. The energy

TABLE IV. The values of the fitting parameters.

\begin{tabular}{lcccc}
\hline \hline$\alpha \alpha^{\prime} \mu$ & $a$ & $b$ & $c$ & $d$ \\
\hline$d p \sigma$ & 118.1935 & -28.6982 & 1.2701 & 1 \\
$d p \pi$ & 513.051 & -158.0415 & 9.6802 & 1 \\
$d d \sigma$ & 120.9216 & -46.1969 & 4.2375 & 1 \\
$d d \pi$ & -274.1753 & 93.9890 & -7.788 & 1 \\
$d d \delta$ & -70.9924 & 21.0086 & -1.3686 & 1 \\
\hline \hline
\end{tabular}




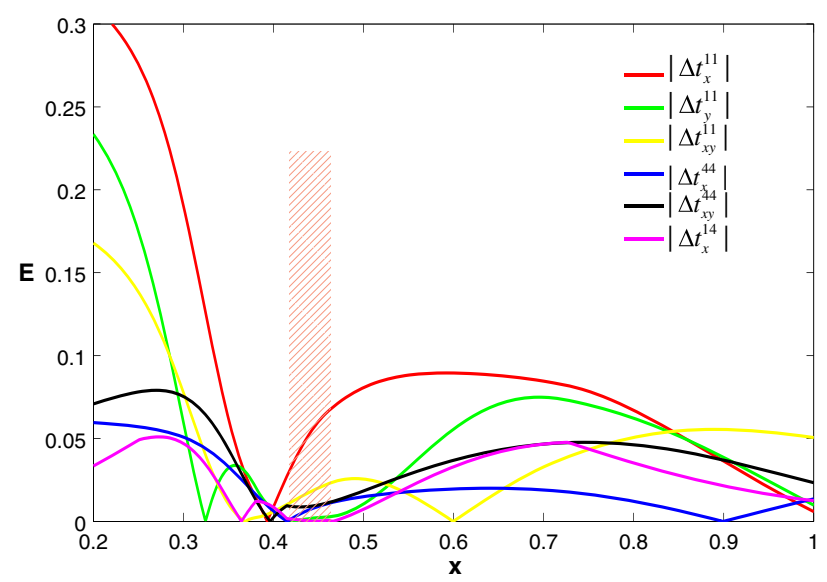

FIG. 10. The changes of hoppings as a function as $x$. Energy is in Rydberg units.

is in Rydberg units. The fitting parameters $a_{\alpha \alpha^{\prime} \mu}, b_{\alpha \alpha^{\prime} \mu}$, $c_{\alpha \alpha^{\prime} \mu}$, and $d_{\alpha \alpha^{\prime} \mu}$ are listed in Table IV.

The effective hoppings in Eqs. (B27)-(B32) can be calculated with the above parameters. The evolution of the hopping difference between the single-layer case and the bulk case as a function of $x$ is shown in Fig. 10. The bulk case has $x_{b}=0.4838$. For the single-layer case, $x$ is generally smaller than $x_{b}$ and usually is located at the shaded rectangle region. We can find that the $t_{x}^{11}$ are strongly affected by the lattice distortion, and other hoppings are weakly adjusted. This is consistent with the artificial parameter used in our paper. In conclusion, we give a clear picture about the electronic structure of single-layer FeSe.

\section{APPENDIX C: EFFECTIVE HAMILTONIAN AROUND M POINTS}

In this section, we present a detailed derivation about the effective Hamiltonian around $\mathrm{M}$ points. The total Hamiltonian is

$$
H=H_{t}+H_{s o}+H_{s} \text {. }
$$

In momentum space,

$$
H=\sum_{\tilde{k}} \bar{\Psi}^{\dagger}(\tilde{k}) \bar{H}(\tilde{k}) \bar{\Psi}(\tilde{k}) .
$$

Here, we set the basis as $\bar{\Psi}(\tilde{k})=\left[\bar{\phi}_{\uparrow}(k), \bar{\phi}_{\uparrow}\left(k^{\prime}\right), \bar{\phi}_{\downarrow}(k)\right.$, $\left.\bar{\phi}_{\downarrow}\left(k^{\prime}\right)\right]^{t} \quad$ with $\quad \bar{\phi}_{\sigma}(\tilde{k})=\left[d_{1, \sigma}(\tilde{k}), d_{2, \sigma}(\tilde{k}), d_{3, \sigma}(\tilde{k}), d_{4, \sigma}(\tilde{k})\right.$, $\left.d_{5, \sigma}(\tilde{k})\right]$. The Hamiltonian kernel $\bar{H}(\tilde{k})$ has the form

$$
\bar{H}(\tilde{k})=\left[\begin{array}{cccc}
A(k)+H_{s o 1}(k) & H_{s}(k) & 0 & H_{s o 2}(k) \\
H_{s}^{\dagger}(k) & A(k)+H_{s o 1}(k) & H_{s o 2}\left(k^{\prime}\right) & 0 \\
0 & H_{s o 2}^{\dagger}\left(k^{\prime}\right) & A\left(k^{\prime}\right)-H_{s o 1}\left(k^{\prime}\right) & H_{s}(k) \\
H_{s o 2}^{\dagger}(k) & 0 & H_{s}(k) & A\left(k^{\prime}\right)-H_{s o 1}\left(k^{\prime}\right)
\end{array}\right] .
$$

The three orbital matrices $\ell^{x, y, z}$ under the basis $\{|x z\rangle,|y z\rangle$, $|x y\rangle\}$ are

$$
\begin{array}{ll}
\ell^{z} & =\left[\begin{array}{ccc}
0 & -i & 0 \\
i & 0 & 0 \\
0 & 0 & 0
\end{array}\right], \quad \ell^{x}=\left[\begin{array}{ccc}
0 & 0 & i \\
0 & 0 & 0 \\
-i & 0 & 0
\end{array}\right], \\
\ell^{y} & =\left[\begin{array}{ccc}
0 & 0 & 0 \\
0 & 0 & i \\
0 & -i & 0
\end{array}\right] .
\end{array}
$$

Then, we can get the explicit forms for $H_{s}(\tilde{k}) H_{s o 1}(\tilde{k})$ and $H_{s o 2}(\tilde{k})$ as

$$
H_{s}(\tilde{k})=\left[\begin{array}{ccccc}
\xi_{s} & 0 & 0 & 0 & 0 \\
0 & \xi_{s} & 0 & 0 & 0 \\
0 & 0 & 0 & 0 & 0 \\
0 & 0 & 0 & \xi_{s} & 0 \\
0 & 0 & 0 & 0 & 0
\end{array}\right]
$$

Now, we define the new basis for $d_{x z, \sigma}(\tilde{k})$ and $d_{y z, \sigma}(\tilde{k})$ orbitals according to the eigenstates of azimuthal and magnetic quantum numbers: $l=2, m= \pm 1$. Namely, 


$$
\begin{aligned}
d_{(2,1), \sigma}(\tilde{k}) & =-\frac{1}{\sqrt{2}}\left[d_{1, \sigma}(\tilde{k})+i d_{2, \sigma}(\tilde{k})\right] \\
d_{(2,-1), \sigma}(\tilde{k}) & =\frac{1}{\sqrt{2}}\left[d_{1, \sigma}(\tilde{k})-i d_{2, \sigma}(\tilde{k})\right] .
\end{aligned}
$$

$\left.d_{4, \sigma}(\tilde{k}), d_{5, \sigma}(\tilde{k})\right]$, the total Hamiltonian can be rewritten as follows:

$$
H=\sum_{\tilde{k}} \Psi^{\dagger}(\tilde{k}) H(\tilde{k}) \Psi(\tilde{k})
$$

Then, in this new basis, $\Psi(\tilde{k})=\left[\phi_{\uparrow}(k), \phi_{\uparrow}\left(k^{\prime}\right), \phi_{\downarrow}(k)\right.$, $\left.\phi_{\downarrow}\left(k^{\prime}\right)\right]^{t} \quad$ with $\quad \phi_{\sigma}(\tilde{k})=\left[d_{(2,1), \sigma}(\tilde{k}), d_{(2,-1), \sigma}(\tilde{k}), d_{3, \sigma}(\tilde{k})\right.$,

Here,

$$
\begin{gathered}
H(\tilde{k})=\left[\begin{array}{cccc}
\tilde{A}(k)+\tilde{H}_{s o 1}(k) & \tilde{H}_{s}(k) & 0 & \tilde{H}_{s o 2}(k) \\
\tilde{H}_{s}^{\dagger}(k) & \tilde{A}(k)+\tilde{H}_{s o 1}(k) & \tilde{H}_{s o 2}\left(k^{\prime}\right) & 0 \\
0 & \tilde{H}_{s o 2}^{\dagger}\left(k^{\prime}\right) & \tilde{A}\left(k^{\prime}\right)-\tilde{H}_{s o 1}\left(k^{\prime}\right) & \tilde{H}_{s}(k) \\
\tilde{H}_{s o 2}^{\dagger}(k) & 0 & \tilde{H}_{s}(k) & \tilde{A}\left(k^{\prime}\right)-\tilde{H}_{s o 1}\left(k^{\prime}\right)
\end{array}\right], \\
\tilde{A}(\tilde{k})=\left[\begin{array}{ccccc}
\tilde{A}_{11}(\tilde{k}) & \tilde{A}_{12}(\tilde{k}) & -\frac{A_{13}(\tilde{k})+i A_{23}(\tilde{k})}{\sqrt{2}} & -\frac{A_{14}(\tilde{k})+i A_{24}(\tilde{k})}{\sqrt{2}} & -\frac{A_{15}(\tilde{k})+i A_{25}(\tilde{k})}{\sqrt{2}} \\
& \tilde{A}_{22}(\tilde{k}) & \frac{A_{13}(\tilde{k})-i A_{23}(\tilde{k})}{\sqrt{2}} & \frac{A_{14}(\tilde{k})-i A_{24}(\tilde{k})}{\sqrt{2}} & \frac{A_{15}(\tilde{k})-i A_{25}(\tilde{k})}{\sqrt{2}} \\
& & A_{33}(\tilde{k}) & A_{34}(\tilde{k}) & A_{35}(\tilde{k}) \\
& & & A_{44}(\tilde{k}) & A_{45}(\tilde{k})
\end{array}\right],
\end{gathered}
$$

with

$$
\begin{aligned}
\tilde{A}_{11 / 22}(\tilde{k}) & =\frac{A_{11}(\tilde{k})+A_{22}(\tilde{k})}{2} \mp i \frac{A_{12}(\tilde{k})-A_{21}(\tilde{k})}{2} \\
\tilde{A}_{12}(\tilde{k}) & =\frac{-A_{11}(\tilde{k})+A_{22}(\tilde{k})}{2}-i \frac{A_{12}(\tilde{k})+A_{21}(\tilde{k})}{2},
\end{aligned}
$$$$
\tilde{H}_{s o 2}(\tilde{k})=\left[\begin{array}{ccccc}
0 & 0 & 0 & -i \lambda_{\|,+}(\tilde{k}) & 0 \\
0 & 0 & 0 & i \lambda_{\|,-}(\tilde{k}) & 0 \\
0 & 0 & 0 & 0 & 0 \\
i \lambda_{\|,-}(\tilde{k}) & -i \lambda_{\|,+}(\tilde{k}) & 0 & 0 & 0 \\
0 & 0 & 0 & 0 & 0
\end{array}\right] \text {, }
$$

where

$$
\tilde{H}_{s}(\tilde{k})=\left[\begin{array}{ccccc}
\xi_{s} & 0 & 0 & 0 & 0 \\
0 & \xi_{s} & 0 & 0 & 0 \\
0 & 0 & 0 & 0 & 0 \\
0 & 0 & 0 & \xi_{s} & 0 \\
0 & 0 & 0 & 0 & 0
\end{array}\right]
$$

$$
\tilde{H}_{s o 1}(\tilde{k})=\left[\begin{array}{ccccc}
-\lambda_{\perp}(\tilde{k}) & 0 & 0 & 0 & 0 \\
0 & \lambda_{\perp}(\tilde{k}) & 0 & 0 & 0 \\
0 & 0 & 0 & 0 & 0 \\
0 & 0 & 0 & 0 & 0 \\
0 & 0 & 0 & 0 & 0
\end{array}\right]
$$

$$
\begin{aligned}
& \lambda_{\|,+}(\tilde{k})=\frac{\left[\lambda_{\|, x z}(\tilde{k})+\lambda_{\|, y z}(\tilde{k})\right]}{\sqrt{2}} \\
& \lambda_{\|,-}(\tilde{k})=\frac{\left[\lambda_{\|, x z}(\tilde{k})-\lambda_{\|, y z}(\tilde{k})\right]}{\sqrt{2}} .
\end{aligned}
$$

From Eq. (C14), we find that the $d_{(2,1), \sigma}(\tilde{k}), d_{(2,-1), \sigma}(\tilde{k})$ can be split by the nonzero $\lambda_{\perp}(\tilde{k})$. If the splitting between them is large enough, we can only keep $d_{(2,-1 / 1), \uparrow / \downarrow}(\tilde{k})$ for $\lambda_{\perp}(\tilde{k})>0$ and $d_{(2,1 /-1), \uparrow / \downarrow}(\tilde{k})$ for $\lambda_{\perp}(\tilde{k})<0$ around the $\mathrm{M}$ point. Hence, we get two kinds of effective Hamiltonians around the $\mathrm{M}$ point, $\lambda_{\perp}(\tilde{k})>0$ and $\lambda_{\perp}(\tilde{k})<0$.

We set the effective basis $\Psi_{\text {eff }}(\tilde{k})=\left[\phi_{\text {eff }, \uparrow}(k)\right.$, $\left.\phi_{\mathrm{eff}, \uparrow}\left(k^{\prime}\right), \phi_{\mathrm{eff}, \downarrow}(k), \phi_{\mathrm{eff}, \downarrow}\left(k^{\prime}\right)\right]^{t} \quad$ with $\quad \phi_{\sigma}(\tilde{k})=\left[d_{x y, \sigma}(\tilde{k})\right.$, $\left.d_{\left(2, \mp(-1)^{\sigma}, \sigma\right.}(\tilde{k})\right]$, and $\sigma=\mp$ for spin $\uparrow$ or $\downarrow$. The effective Hamiltonian in this basis is 


$$
H_{\mathrm{eff}}=\sum_{\tilde{k}} \Psi_{\mathrm{eff}}^{\dagger}(\tilde{k}) H_{\mathrm{eff}}(\tilde{k}) \Psi_{\mathrm{eff}}(\tilde{k})
$$

with

$$
H_{\mathrm{eff}}(\tilde{k})=\left[\begin{array}{ll}
H_{\mathrm{eff}, 11}(\tilde{k}) & H_{\mathrm{eff}, 12}(\tilde{k}) \\
H_{\mathrm{eff}, 12}^{\dagger}(\tilde{k}) & H_{\mathrm{eff}, 22}(\tilde{k})
\end{array}\right],
$$

where

$$
\begin{aligned}
& H_{\mathrm{eff}, 11}=\left[\begin{array}{cccc}
A_{44}(k) & \pm \frac{A_{41}(k) \pm i A_{42}(k)}{\sqrt{2}} & \xi_{s} & 0 \\
& \frac{A_{11}(k)+A_{22}(k)}{2}+\left|\lambda_{\perp}(k)\right| & 0 & \xi_{s} \\
& & A_{44}\left(k^{\prime}\right) & \pm \frac{A_{41}\left(k^{\prime}\right) \pm i A_{42}\left(k^{\prime}\right)}{\sqrt{2}} \\
& & & \frac{A_{11}\left(k^{\prime}\right)+A_{22}\left(k^{\prime}\right)}{2}+\left|\lambda_{\perp}\left(k^{\prime}\right)\right|
\end{array}\right] \\
& H_{\mathrm{eff}, 22}=\left[\begin{array}{cccc}
A_{44}(k) & \mp \frac{A_{41}(k) \mp i A_{42}(k)}{\sqrt{2}} & \xi_{s} & 0 \\
& \frac{A_{11}(k)+A_{22}(k)}{2}+\left|\lambda_{\perp}(k)\right| & 0 & \xi_{s} \\
& & A_{44}\left(k^{\prime}\right) & \mp \frac{A_{41}\left(k^{\prime}\right) \mp i A_{42}\left(k^{\prime}\right)}{\sqrt{2}} \\
& & & \frac{A_{11}\left(k^{\prime}\right)+A_{22}\left(k^{\prime}\right)}{2}+\left|\lambda_{\perp}(k)\right|
\end{array}\right] \text {, } \\
& H_{\mathrm{eff}, 12}(\tilde{k})=\left[\begin{array}{cccc}
0 & 0 & 0 & \pm i \frac{\left[\lambda_{\|, x z}(k) \mp \lambda_{\|, y z}(k)\right]}{\sqrt{2}} \\
0 & 0 & \pm i \frac{\left[\lambda_{\|, x z}(k) \mp \lambda_{\|, y z}(k)\right]}{\sqrt{2}} & 0 \\
0 & \pm i \frac{\left[\lambda_{\|, x z}\left(k^{\prime}\right) \mp \lambda_{\|, y z}\left(k^{\prime}\right)\right]}{\sqrt{2}} & 0 & 0 \\
\pm i \frac{\left[\lambda_{\|, x z}\left(k^{\prime}\right) \mp \lambda_{\|, y z}\left(k^{\prime}\right)\right]}{\sqrt{2}} & 0 & 0 & 0
\end{array}\right] .
\end{aligned}
$$

When all the parity-mixed terms are zero, in the basis $\left[d_{x y, \uparrow}(\tilde{k}), d_{(2, \mp 1), \uparrow}(\tilde{k}), d_{x y, \downarrow}(\tilde{k}), d_{(2, \pm 1), \downarrow}(\tilde{k})\right]$, we get the effective Hamiltonian around the M point as

$$
H_{\mathrm{eff}}(\tilde{k})=\left[\begin{array}{cccc}
A_{44}(\tilde{k}) & h_{12}(\tilde{k}) & 0 & 0 \\
& h_{22}(\tilde{k}) & 0 & 0 \\
& & A_{44}(\tilde{k}) & h_{12}^{*}(\tilde{k}) \\
& & & h_{22}(\tilde{k})
\end{array}\right],
$$

with

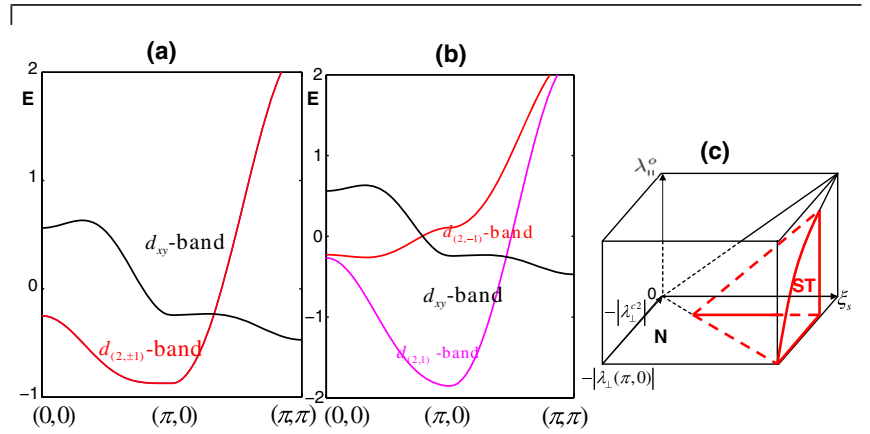

FIG. 11. Panels (a) and (b) show the band inversion that occurs around $\mathrm{M}$ point from the normal phase (a) with $\lambda_{\perp}^{o}=0.0$, $\lambda_{\perp}^{n n}=0.0, \lambda_{\|}^{o}=0.0$, and $\xi_{s}=0$, to a topological phase with $\lambda_{\perp}^{o}=$ $0.5, \lambda_{\perp}^{n n}=-0.12, \lambda_{\|}^{o}=0.0$, and $\xi_{s}=0$. Here, we show the parity-even part. The parity-odd part has a similar picture. (c) The phase diagram as a function of $\lambda_{\perp}(\pi, 0), \lambda_{\|}^{o}$, and $\xi_{s}$ for $\lambda_{\perp}(\pi, 0)<0$. Here, ST and $\mathrm{N}$ label the strong topological phase and the normal trivial phase. 


$$
\begin{aligned}
h_{12}(\tilde{k}) & =\sqrt{2} t_{x}^{14}\left(-\sin \tilde{k}_{y} \pm i \sin \tilde{k}_{x}\right) \\
h_{22}(\tilde{k}) & =\frac{A_{11}(\tilde{k})+A_{22}(\tilde{k})}{2}+\left|\lambda_{\perp}(\tilde{k})\right| \\
\varepsilon_{0}(\tilde{k}) & =\frac{1}{2}\left\{A_{44}(\tilde{k})+\frac{1}{2}\left[A_{11}(\tilde{k})+A_{22}(\tilde{k})\right]+\left|\lambda_{\perp}(\tilde{k})\right|\right\} \\
\varepsilon_{1}(\tilde{k}) & =\frac{1}{2}\left\{A_{44}(\tilde{k})-\frac{1}{2}\left[A_{11}(\tilde{k})+A_{22}(\tilde{k})\right]-\left|\lambda_{\perp}(\tilde{k})\right|\right\} \\
\varepsilon_{12}(\tilde{k}) & =\sqrt{2} t_{x}^{14} \sin \tilde{k}_{y}, \quad \varepsilon_{13}(\tilde{k})=\mp \sqrt{2} t_{x}^{14} \sin \tilde{k}_{x},
\end{aligned}
$$

when $\lambda_{\perp}(k)>0$ and all the parity-mixed terms are zero. We consider the effective Hamiltonian shown in Eq. (C21); the picture of the band inversion induced by the spin-orbital coupling is shown in Figs. 11(a) and 11(b).

When $\lambda_{\perp}(k)<0$ and all the parity-mixed terms are nonzero, we consider the effective Hamiltonian Eq. (C16); the spectrum is

$E(k)= \pm \sqrt{\varepsilon_{12}^{2}(k)+\varepsilon_{13}^{2}(k)+\left[\sqrt{\varepsilon_{1}^{2}(k)+2\left|\lambda_{\|}^{o}\right|^{2}} \pm \xi_{s}\right]}$.

The phase boundary is determined by the condition

$$
\varepsilon_{1}^{2}(k)+2\left|\lambda_{\|}^{o}\right|^{2}=\xi_{s}^{2} .
$$

According to Eq. (C24), we plot the phase diagram that is shown in Fig. 11(c).

[1] Y. Kamihara, T. Watanabe, M. Hirano, and H. Hosono, Iron-Based Layered Superconductor $\mathrm{La}\left[\mathrm{O}_{1-x} \mathrm{~F}_{x}\right] \mathrm{FeAs}$ $(x=0.05-0.12)$ with $T_{c}=26 \mathrm{~K}$, J. Am. Chem. Soc. 130, 3296 (2008).

[2] X. L. Qi and S. C. Zhang, Topological Insulators and Superconductors, Rev. Mod. Phys. 83, 1057 (2011).

[3] M.Z. Hasan and C.L. Kane, Colloquium: Topological Insulators, Rev. Mod. Phys. 82, 3045 (2010).

[4] Q. Y. Wang, Z. Li, W. H. Zhang, Z. C. Zhang, J. S. Zhang, W. Li, H. Ding, Y. B. Ou, P. Deng, K. Chang et al., Interface Induced High Temperature Superconductivity in Single Unit-Cell FeSe Films on $\mathrm{SrTiO}_{3}$, Chin. Phys. Lett. 29, 037402 (2012).

[5] D. F. Liu, W. H. Zhang, D. X. Mou, J. F. He, Y. B. Ou, Q. Y. Wang, Z. Li, L. L. Wang, L. Zhao, S. L. He et al., Electronic Origin of High Temperature Superconductivity in Single-Layer FeSe Superconductor, Nat. Commun. 3, 931 (2012).

[6] S. L. He, J. F. He, W. H. Zhang, L. Zhao, D. F. Liu, X. Liu, D. X. Mou, Y. B. Ou, Q. Y. Wang, Z. Li et al., Phase Diagram and High Temperature Superconductivity at $65 \mathrm{~K}$ in Tuning Carrier Concentration of Single-Layer FeSe Films, Nat. Mater. 12, 605 (2013).
[7] S. Y. Tan, Y. Zhang, M. Xia, Z. R. Ye, F. Chen, X. Xie, R. Peng, D. F. Xu, Q. Fan, H. C. Xu et al., Interface-Induced Superconductivity and Strain-Dependent Spin Density Waves in $\mathrm{FeSe} / \mathrm{SrTiO}_{3}$ Thin Films, Nat. Mater. 12, 634 (2013).

[8] R. Peng, H. C. Xu, S. Y. Tan, M. Xia, X. P. Shen, Z. C. Huang, C. H. P. Wen, Q. Song, T. Zhang, B. P. Xie et al., Critical Role of Substrate in the High Temperature Superconductivity of Single Layer FeSe on $\mathrm{Nb}_{\mathrm{BaTiO}}$, arXiv:1402.1357 [Phys. Rev. X (to be published)].

[9] W. H. Zhang, Y. Sun, J. S. Zhang, F. S. Li, M. H. Guo, Y. F. Zhao, H. M. Zhang, J. P. Peng, Y. Xing, H. C. Wang et al., Direct Observation of High-Temperature Superconductivity in One-Unit-Cell FeSe Films, Chin. Phys. Lett. 31, 017401 (2014).

[10] J. J. Lee, F. T. Schmitt, R. G. Moore, S. Johnston, Y. T. Cui, W. Li, M. Yi, Z. K. Liu, M. Hashimoto, Y. Zhang et al., Significant $T_{c}$ Enhancement in FeSe Films on $\mathrm{SrTiO}_{3}$ Due to Interfacial Mode Coupling, arXiv:1312.2633.

[11] F. C. Hsu, J. Y. Luo, K. W. Yeh, T. K. Chen, T. W. Huang, P. M. Wu, Y. C. Lee, Y. L. Huang, Y. Y. Chu, D. C. Yan et al., Superconductivity in the PbO-Type Structure Alpha$\mathrm{FeSe}$, Proc. Natl. Acad. Sci. U.S.A. 105, 14262 (2008).

[12] K. W. Yeh, T. W. Huang, Y. L. Huang, T. K. Chen, F. C. Hsu, P. M. Wu, Y. C. Lee, Y. Y. Chu, C. L. Chen, J. Y. Luo et al., Tellurium Substitution Effect on Superconductivity of the Alpha-Phase Iron Selenide, Europhys. Lett. 84, 37002 (2008).

[13] D. C. Johnston, The Puzzle of High Temperature Superconductivity in Layered Iron Pnictides and Chalcogenides, Adv. Phys. 59, 803 (2010).

[14] E. Dagotto, Colloquium: The Unexpected Properties of Alkali Metal Iron Selenide Superconductors, Rev. Mod. Phys. 85, 849 (2013).

[15] A. Subedi, L. Zhang, D. J. Singh, and M. H. Du, Density Functional Study of FeS, FeSe, and FeTe: Electronic Structure, Magnetism, Phonons, and Superconductivity, Phys. Rev. B 78, 134514 (2008).

[16] P. Richard, T. Sato, K. Nakayama, T. Takahashi, and H. Ding, Fe-Based Superconductors: An Angle-Resolved Photoemission Spectroscopy Perspective, Rep. Prog. Phys. 74, 124512 (2011).

[17] K. Kuroki, S. Onari, R. Arita, H. Usui, Y. Tanaka, H. Kontani, and H. Aoki, Unconventional Pairing Originating from the Disconnected Fermi Surfaces of Superconducting $\mathrm{LaFeAsO}_{1-x} \mathrm{~F}_{x}$, Phys. Rev. Lett. 101, 087004 (2008).

[18] S. Graser, T. A. Maier, P. J. Hirschfeld, and D. J. Scalapino, Near-Degeneracy of Several Pairing Channels in Multiorbital Models for the Fe-Pnictides, New J. Phys. 11, 025016 (2009).

[19] J. Hu and N. Hao, $S_{4}$ Symmetric Microscopic Model for Iron-Based Superconductors, Phys. Rev. X 2, 021009 (2012).

[20] J. Hu, Iron-Based Superconductors as Odd-Parity Superconductors, Phys. Rev. X 3, 031004 (2013).

[21] V. Cvetkovic and O. Vafek, Space Group Symmetry, Spin-Orbit Coupling, and the Low-Energy Effective Hamiltonian for Iron-Based Superconductors, Phys. Rev. B 88, 134510 (2013). 
[22] J. C. Slater and G. F. Koster, Simplified LCAO Method for the Periodic Potential Problem, Phys. Rev. 94, 1498 (1954).

[23] P. A. Lee and X.-G. Wen, Spin-Triplet p-Wave Pairing in a Three-Orbital Model for Iron Pnictide Superconductors, Phys. Rev. B 78, 144517 (2008).

[24] C. L. Kane and E. J. Mele, $Z_{2}$ Topological Order and the Quantum Spin Hall Effect, Phys. Rev. Lett. 95, 146802 (2005).

[25] B. A. Bernevig, T. L. Hughes, and S.-C. Zhang, Quantum Spin Hall Effect and Topological Phase Transition in HgTe Quantum Wells, Science 314, 1757 (2006).

[26] L. Fu and C. L. Kane, Topological Insulators with Inversion Symmetry, Phys. Rev. B 76, 045302 (2007).

[27] L. Fu, Topological Crystalline Insulators, Phys. Rev. Lett. 106, 106802 (2011).

[28] T. H. Hsieh, H. Lin, J. Liu, W. Duan, A. Bansil, and L. Fu, Topological Crystalline Insulators in the SnTe Material Class, Nat. Commun. 3, 982 (2012).

[29] C.-X. Liu, R.-X. Zhang, and B. K. Vanleeuwen, Topological Non-symmorphic Crystalline Insulators, Phys. Rev. B 90, 085304 (2014).

[30] K. Liu, Z. Lu, and T. Xiang, Atomic and Electronic Structures of FeSe Monolayer and Bilayer Thin Films on $\mathrm{SrTiO}_{3}(001)$ : First-Principles Study, Phys. Rev. B 85, 235123 (2012).

[31] T. Bazhirov and M. L. Cohen, Effects of Charge Doping and Constrained Magnetization on the Electronic Structure of an FeSe Monolayer, J. Phys. Condens. Matter 25, 105506 (2013).

[32] F. Zheng, Z. Wang, W. Kang, and P. Zhang, Antiferromagnetic FeSe Monolayer on $\mathrm{SiTiO}_{3}$ : The Charge Doping and Electric Field Effects, Sci. Rep. 3, 2213 (2013).

[33] M. L. Tiago, Y. Zhou, M. M. G. Alemany, Y. Saad, and J. R. Chelikowsky, Evolution of Magnetism in Iron from the Atom to the Bulk, Phys. Rev. Lett. 97, 147201 (2006).

[34] A. Kreisel, Y. Wang, T. A. Maier, P. J.Hirschfeld, and D. J. Scalapino, Spin Fluctuations and Superconductivity in $\mathrm{K}_{x} \mathrm{Fe}_{2-y} \mathrm{Se}_{2}$, Phys. Rev. B 88, 094522 (2013).

[35] R. Peng, X. P. Shen, X. Xie, H. C. Xu, S. Y. Tan, M. Xia, T. Zhang, H. Y. Cao, X. G. Gong, J. P. Hu et al., Measurement of an Enhanced Superconducting Phase and a Pronounced Anisotropy of the Energy Gap of a Strained FeSe Single
Layer in $\mathrm{FeSe} / \mathrm{Nb}: \mathrm{SrTiO}_{3} / \mathrm{KTaO}_{3}$ Heterostructures Using Photoemission Spectroscopy, Phys. Rev. Lett. 112, 107001 (2014).

[36] B. Yan, M. Jansen, and C. Felser, A Large Energy-Gap Oxide Topological Insulator Based on the Superconductor $\mathrm{BaBiO}_{3}$, Nat. Phys. 9, 709 (2013).

[37] L. Fu and C. L. Kane, Superconducting Proximity Effect and Majorana Fermions at the Surface of a Topological Insulator, Phys. Rev. Lett. 100, 096407 (2008).

[38] R. M. Lutchyn, J. D. Sau, and S. D. Sarma, Majorana Fermions and a Topological Phase Transition in Semiconductor-Superconductor Heterostructures, Phys. Rev. Lett. 105, 077001 (2010).

[39] L. Fu and E. Berg, Odd-Parity Topological Superconductors: Theory and Application to $\mathrm{CuxBi}_{2} \mathrm{Se}_{3}$, Phys. Rev. Lett. 105, 097001 (2010).

[40] N. Hao and J. Hu, Odd Parity Pairing and Nodeless Antiphase $s^{ \pm}$in Iron-Based Superconductors, Phys. Rev. B 89, 045144 (2014).

[41] M. König, S. Wiedmann, C. Brüne, A. Roth, H. Buhmann, L. W. Molenkamp, X. Qi, and S. Zhang, Quantum Spin Hall Insulator State in HgTe Quantum Wells, Science 318, 766 (2007).

[42] F. Yang, L. Miao, Z. Wang, M. Yao, F. Zhu, Y. Song, M. Wang, J. Xu, A. V. Fedorov, Z. Sun, G. Zhang, C. Liu, F. Liu, D. Qian, C. Gao, and J. Jia, Spatial and Energy Distribution of Topological Edge States in Single Bi(111) Bilayer, Phys. Rev. Lett. 109, 016801 (2012).

[43] S. Kim, K. Jin, J. Park, J. Kim, S. Jhi, T. Kim, and H. Yeom, Edge and Interfacial States in a Two-Dimensional Topological Insulator: Bi(111) Bilayer on $\mathrm{Bi}_{2} \mathrm{Te}_{2} \mathrm{Se}$, Phys. Rev. B 89, 155436 (2014).

[44] I. K. Drozdov, A. Alexandradinata, S. Jeon, S. Nadj-Perge, H. Ji, R. Cava, B. A. Bernevig, and A. Yazdani, OneDimensional Topological Edge States of Bismuth Bilayers, Nat. Phys. 10, 664 (2014).

[45] H. Eschrig and K. Koepernik, Tight-Binding Models for the Iron-Based Superconductors, Phys. Rev. B 80, 104503 (2009).

[46] D. A. Papaconstantopoulos, M. J. Mehl, and M. D. Johannes, Tight-Binding Hamiltonian for LaOFeAs, Phys. Rev. B 82, 054503 (2010). 